\title{
Finite-approximate controllability of fractional stochastic evolution equations with nonlocal conditions
}

\author{
Yonghong Ding ${ }^{1,2^{*}}$ and Yongxiang $\mathrm{Li}^{1}$
}

\section{"Correspondence:}

dyh198510@126.com

'Department of Mathematics,

Northwest Normal University,

Lanzhou, China

${ }^{2}$ Department of Mathematics,

Tianshui Normal University,

Tianshui, China

\section{Springer}

\begin{abstract}
This paper deals with the finite-approximate controllability for a class of fractional stochastic evolution equations with nonlocal initial conditions in a Hilbert space. We establish sufficient conditions for the finite-approximate controllability of the control system when the compactness conditions or Lipschitz conditions for the nonlocal term and uniform boundedness conditions for the nonlinear term are not required. The discussion is based on the fixed point theorem, approximation techniques and diagonal argument. In the end, an example is presented to illustrate the abstract theory. Our result improves and extends some relevant results in this area.
\end{abstract}

MSC: $93 \mathrm{BB} 05 ; 60 \mathrm{H} 15 ; 47 \mathrm{~J} 35$

Keywords: Nonlocal problem; Semigroups; Finite-approximate controllability; Stochastic evolution equations

\section{Introduction}

In this paper, we shall be concerned with the finite-approximate controllability (see Definition 2.8 in Sect. 2) for following fractional evolution equations with nonlocal initial conditions of the form

$$
\left\{\begin{array}{l}
{ }^{c} D_{t}^{\alpha} x(t)+A x(t)=f(t, x(t))+\sigma(t, x(t)) \frac{d W(t)}{d t}+B u(t), \quad t \in[0, b], \\
x(0)=g(x),
\end{array}\right.
$$

where ${ }^{c} D_{t}^{\alpha}$ is the Caputo fractional derivative of order $\frac{1}{2}<\alpha \leq 1$, and

$$
g(x)=\int_{0}^{b} h(s, x(s)) d s
$$

Let $\mathbb{H}, \mathbb{K}$ be two separable Hilbert spaces and the state $x(\cdot)$ takes its values in $\mathbb{H}$. Also $A: D(A) \subset \mathbb{H} \rightarrow \mathbb{H}$ is a closed linear operator and $-A$ is the infinitesimal generator of a $C_{0}$-semigroup $T(t)(t \geq 0)$ on $\mathbb{H}$. For convenience, we will use the same notation $\|\cdot\|$ to denote the norms in $\mathbb{H}$ and $\mathbb{K}$, and $\langle\cdot, \cdot\rangle$ to denote the inner product of $\mathbb{H}$ and $\mathbb{K}$ without any

(c) The Author(s) 2020. This article is licensed under a Creative Commons Attribution 4.0 International License, which permits use, sharing, adaptation, distribution and reproduction in any medium or format, as long as you give appropriate credit to the original author(s) and the source, provide a link to the Creative Commons licence, and indicate if changes were made. The images or other third party material in this article are included in the article's Creative Commons licence, unless indicated otherwise in a credit line to the material. If material is not included in the article's Creative Commons licence and your intended use is not permitted by statutory regulation or exceeds the permitted use, you will need to obtain permission directly from the copyright holder. To view a copy of this licence, visit http://creativecommons.org/licenses/by/4.0/. 
confusion. We are also employing the same notation $\|\cdot\|$ for the norm of $L(\mathbb{K}, \mathbb{H})$, which denotes the space of all bounded linear operators from $\mathbb{K}$ into $\mathbb{H}$. Suppose that $\{W(t): t \geq$ $0\}$ is a $\mathbb{K}$-valued Brownian motion or Wiener process with a finite trace nuclear covariance operator $Q \geq 0$ defined on a filtered complete probability space $\left(\Omega, \mathcal{F},\left\{\mathcal{F}_{t}\right\}_{t \geq 0}, P\right)$. The control function $u(\cdot)$ belongs to the space $L_{\mathcal{F}}^{2}(J, \mathbb{U})$, a Banach space of admissible control functions, for a separable Hilbert space $\mathbb{U}, B: \mathbb{U} \rightarrow \mathbb{H}$ is a bounded linear operator, while $f, \sigma$ and $h$ are appropriate functions to be given later.

It is well known that nonlocal problems have better properties in applications than the classical ones, so differential equations with nonlocal initial conditions have been studied by many authors, see [1-13] and the references therein. However, in order to establish the main results, the assumption of compactness or Lipschitz condition on the nonlocal term plays an important role in these articles. But this restriction is too strong and is not usually satisfied in practical applications.

Recently, Liang, Liu, and Xiao [14] investigated the existence of mild solutions for a class of nonlocal Cauchy problem under the hypothesis that the nonlocal term $g$ satisfies the condition

(H) For any $u, w \in C([0, b] ; E)$, there exists a constant $\delta \in(0, b)$ such that $u(t)=w(t)$ $(t \in[\delta, b])$ implies $g(u)=g(w)$.

Note that assumption $(\mathrm{H})$ is for the case when the values of the solution $x(t)$ for $t$ near zero do not affect $g(x)$. With the help of assumption $(\mathrm{H})$, the authors relaxed the compactness and Lipschitz continuity on the nonlocal item $g$.

On the other hand, controllability for various linear and nonlinear dynamical systems have been considered in many publications by using different approaches due to its applications in many fields of science and engineering, see [15-32]. It should be emphasized that there are many different notions of controllability for dynamical systems, for example, approximate controllability, exact controllability, null controllability, and so on. There have been many papers on the approximate controllability for semilinear evolution systems in abstract spaces, see [16-21,32] and the references therein. Several authors have studied exact controllability for differential control systems, see [22-25] and the references therein. It is worth mentioning that [15] studied simultaneous approximate and finite-dimensional exact controllability (finite-approximate controllability) of the following control system:

$$
\left\{\begin{array}{l}
{ }^{c} D_{t}^{\alpha} x(t)=A x(t)=f(t, x(t))+B u(t), \quad t \in[0, b], \\
x(0)=x_{0}+g(x),
\end{array}\right.
$$

where $f:[0, T] \times X \rightarrow X, g: C([0, T], X) \rightarrow X$, and $X$ is a Hilbert space. In this paper, finiteapproximate controllability means that system (1.3) is approximately controllable in $X$ as well as exactly controllable in a finite dimensional subspace $E \subset X$. The author obtained sufficient conditions for the finite-approximate controllability of system (1.3) when the nonlocal term $g$ satisfies Lipschitz-type conditions and the nonlinear term $f$ satisfies a growth condition.

In recent years, stochastic differential equations have attracted great interest due to their successful applications to problems in mechanics, electricity, economics, physics, and several fields in engineering. For details, see [33-39] and the references therein. In particular, some researchers investigated controllability of stochastic dynamical control systems 
in infinite-dimensional spaces, see [26-31]. However, there are few works that have reported about the study of controllability problems for stochastic evolution equations with nonlocal conditions, see [30,31], and the authors suppose that the nonlocal item $g$ is a completely continuous map in these papers.

Inspired by the above discussions, especially [15], in this work, we will study the finite-approximate controllability for (1.1). The first novelty of this article is the finiteapproximate controllability which is a stronger version of the controllability concept. Up to now, no one has studied the finite-approximate controllability for a stochastic system, this paper fills this gap in the literature. The second novelty of this article is that the nonlocal term $g(x)$ defined by (1.2) depends on all values of $x$ on the whole interval $[0, b]$, so the methods used in [14] are not valid for the present paper. By using stochastic analysis, approximation techniques, diagonal argument, and Schauder fixed-point theorem, the finite-approximate controllability results are established under weaker conditions in which $g(x)$ is not necessarily Lipschitz continuous or has some compactness property. More precisely, the nonlocal term $g(x)$ depends on all the values of $x$ in the whole interval $[0, b]$, is only continuous and satisfies some weak growth condition. The third novelty of this article is that in almost all the articles on the topic of approximate controllability, for example, see [16-21, 28-31], the authors always require the nonlinear term $f$ be uniformly bounded. In the present work we delete this restriction, and only need the nonlinear term which satisfies some natural growth conditions. So the theorems obtained here extend and complement those obtained in [16-21, 28-31]. In addition, as a special case, the methods used in the present paper can be applied to study the finite-approximate controllability of deterministic systems with nonlocal conditions by suitably introducing the abstract space and norm. The corresponding results that appear are also new.

We organize the paper in the following way: In Sect. 2, we introduce some useful definitions and preliminary results to be used in this paper. In Sect. 3, we state and prove finite-approximate controllability results for fractional stochastic evolution equation with nonlocal conditions. Finally, in Sect. 4, an example is provided to illustrate the applications of the obtained results.

\section{Preliminaries}

In this section, we introduce notations, definitions, and preliminary facts which are used throughout this article.

Let $\left(\Omega, \mathcal{F},\left\{\mathcal{F}_{t}\right\}_{t \geq 0}, P\right)$ be a filtered complete probability space satisfying the usual conditions, which means that the filtration is a right-continuous increasing family and $\mathcal{F}_{0}$ contains all $P$-null sets. Let $\left\{e_{k}, k \in \mathbb{N}\right\}$ be a complete orthonormal basis of $\mathbb{K}$. We denote by $\{W(t): t \geq 0\}$ a cylindrical $\mathbb{K}$-valued Brownian motion or Wiener process defined on the probability space $\left(\Omega, \mathcal{F},\left\{\mathcal{F}_{t}\right\}_{t \geq 0}, P\right)$ with a finite trace nuclear covariance operator $Q \geq 0$, and we let $\operatorname{Tr}(Q)=\sum_{k=1}^{\infty} \lambda_{k}=\lambda<\infty$, which implies that $Q e_{k}=\lambda_{k} e_{k}, k \in \mathbb{N}$. Let $\left\{W_{k}(t), k \in \mathbb{N}\right\}$ be a sequence of one-dimensional standard Wiener processes mutually independent on $\left(\Omega, \mathcal{F},\left\{\mathcal{F}_{t}\right\}_{t \geq 0}, P\right)$ such that

$$
W(t)=\sum_{k=1}^{\infty} \sqrt{\lambda_{k}} W_{k}(t) e_{k}, \quad t \geq 0 .
$$

Furthermore, we assume that $\mathcal{F}_{t}=\sigma\{W(s), 0 \leq s \leq t\}$ is the $\sigma$-algebra generated by $W$ and $\mathcal{F}_{b}=\mathcal{F}$. 
Let $L_{2}^{0}=L_{2}\left(Q^{\frac{1}{2}} \mathbb{K}, \mathbb{H}\right)$ denote the space of all Hilbert-Schmidt operators from $Q^{\frac{1}{2}} \mathbb{K}$ into $\mathbb{H}$ with the inner product $\langle\phi, \varphi\rangle=\operatorname{Tr}\left(\phi Q \varphi^{*}\right)$. It also turns out to be a separable Hilbert space. The collection of all $\mathcal{F}_{b}$-measurable, square-integrable $\mathbb{H}$-valued random variables, denoted $L^{2}(\Omega, \mathbb{H})$, is a Banach space equipped with the norm $\|x\|_{L^{2}}=$ $\left(\mathbb{E}\|x(\omega)\|^{2}\right)^{\frac{1}{2}}$, where $\mathbb{E}$ denotes the expectation with respect to the measure $P$. Let $C\left([0, b], L^{2}(\Omega, \mathbb{H})\right)$ be the Banach space of all continuous mappings from $[0, b]$ to $L^{2}(\Omega, \mathbb{H})$ satisfying $\sup _{t \in[0, b]}\left(\mathbb{E}\|x(t)\|^{2}\right)<\infty$. We use $\mathcal{H}\left([0, b], L^{2}(\Omega, \mathbb{H})\right)$ to denote the space of all $\mathcal{F}_{t}$-adapted measurable processes $x \in C\left([0, b], L^{2}(\Omega, \mathbb{H})\right)$ endowed with the norm $\|x\|_{\mathcal{H}}=$ $\left(\sup _{t \in[0, b]} \mathbb{E}\|x(t)\|^{2}\right)^{\frac{1}{2}}$. The theory of stochastic integrals in Hilbert space can be found in $[37,39]$.

In the rest of the manuscript, we suppose that $A$ generates a compact $C_{0}$-semigroup $T(t)(t \geq 0)$ of uniformly bounded linear operators in $\mathbb{H}$. That is, there exists a positive constant $M \geq 1$ such that $\|T(t)\| \leq M$ for all $t \geq 0$. For any constant $r>0$, let $B_{r}=\left\{x \in \mathcal{H}\left([0, b], L^{2}(\Omega, \mathbb{H})\right):\|x\|_{\mathcal{H}}^{2} \leq r\right\}$. Evidently, $B_{r}$ is a bounded closed convex set in $\mathcal{H}\left([0, b], L^{2}(\Omega, \mathbb{H})\right)$.

By [40, Proposition 2.8], we have the following result which will be used throughout this paper.

Lemma 2.1 If $h:[0, b] \times \mathbb{H} \rightarrow L(\mathbb{K}, \mathbb{H})$ is continuous and $x \in C\left([0, b], L^{2}(\Omega, \mathbb{H})\right)$, then

$$
\mathbb{E}\left\|\int_{[0, b]} h(t, x(t)) d W(t)\right\|^{2} \leq \operatorname{Tr}(Q) \int_{[0, b]} \mathbb{E}\|h(t, x(t))\|^{2} d t
$$

Definition 2.2 ([41]) The Riemann-Liouville fractional integral of order $\alpha>0$ of a function $y:(0,+\infty) \rightarrow \mathbb{R}$ is given by

$$
I_{0}^{\alpha} y(t)=\frac{1}{\Gamma(\alpha)} \int_{0}^{t}(t-s)^{\alpha-1} y(s) d s
$$

provided the right-hand side is pointwise defined on $(0,+\infty)$.

Definition 2.3 ([41]) The Riemann-Liouville fractional derivative of order $\alpha>0$ of a function $y:[0,+\infty) \rightarrow \mathbb{R}$ is given by

$$
D_{0}^{\alpha} y(t)=\frac{1}{\Gamma(n-\alpha)}\left(\frac{d}{d t}\right)^{n} \int_{0}^{t} \frac{y(s)}{(t-s)^{\alpha-n+1}} d s
$$

where $n=[\alpha]+1$, provided that the right-hand side is pointwise defined on $(0,+\infty)$.

Definition 2.4 ([41]) The Caputo fractional derivative of order $\alpha>0$ of a function $y$ : $[0,+\infty) \rightarrow \mathbb{R}$ is given by

$$
{ }^{c} D_{0}^{\alpha} y(t)=D_{0}^{\alpha}\left[y(t)-\sum_{k=0}^{n-1} \frac{t^{k}}{k !} y^{(k)}(0)\right],
$$

where $n=[\alpha]+1$, provided that the right-hand side is pointwise defined on $(0,+\infty)$. 


\section{Remark 2.5}

(i) If $y(t) \in C^{n}[0,+\infty)$, then

$$
{ }^{c} D_{0}^{\alpha} y(t)=\frac{1}{\Gamma(n-\alpha)} \int_{0}^{t} \frac{y^{(n)}(s)}{(t-s)^{\alpha-n+1}} d s=I_{0}^{n-\alpha} y^{(n)}(t)
$$

(ii) If $y(t)$ is an abstract function with values in $E$, then the integrals which appear in Definition 2.2, 2.3 and 2.4 are taken in Bochner's sense.

(iii) The Caputo derivative of a constant is equal to zero.

For $x \in \mathbb{H}$, we define two operators $\mathcal{T}_{\alpha}(t)(t \geq 0)$ and $\mathcal{S}_{\alpha}(t)(t \geq 0)$ as follows:

$$
\mathcal{T}_{\alpha}(t) x=\int_{0}^{\infty} \zeta_{\alpha}(\theta) T\left(t^{\alpha} \theta\right) x d \theta, \quad \mathcal{S}_{\alpha}(t) x=\alpha \int_{0}^{\infty} \theta \zeta_{\alpha}(\theta) T\left(t^{\alpha} \theta\right) x d \theta
$$

where

$$
\begin{aligned}
& \zeta_{\alpha}(\theta)=\frac{1}{\alpha} \theta^{-1-1 / \alpha} \rho_{\alpha}\left(\theta^{-1 / \alpha}\right), \\
& \rho_{\alpha}(\theta)=\frac{1}{\pi} \sum_{k=0}^{\infty}(-1)^{n-1} \theta^{-\alpha n-1} \frac{\Gamma(n \alpha+1)}{n !} \sin (n \pi \alpha), \quad \theta \in(0, \infty) .
\end{aligned}
$$

Here $\zeta_{\alpha}(\theta)$ is a probability density function defined on $(0,+\infty)$, that is,

$$
\zeta_{\alpha}(\theta) \geq 0, \theta \in(0, \infty), \quad \int_{0}^{\infty} \zeta_{\alpha}(\theta) d \theta=1, \quad \int_{0}^{\infty} \theta \zeta_{\alpha}(\theta) d \theta=\frac{1}{\Gamma(1+\alpha)}
$$

The following properties of the operators $\mathcal{T}_{\alpha}(t)(t \geq 0)$ and $\mathcal{S}_{\alpha}(t)(t \geq 0)$, which can be found in $[6,7]$, will be needed in our arguments.

Lemma 2.6 The operators $\mathcal{T}_{\alpha}(t)(t \geq 0)$ and $\mathcal{S}_{\alpha}(t)(t \geq 0)$ satisfy the following properties:

(i) For any fixed $t \geq 0, \mathcal{T}_{\alpha}(t)$ and $\mathcal{S}_{\alpha}(t)$ are linear and bounded operators in $\mathbb{H}$, i.e., for any $x \in \mathbb{H}$,

$$
\left\|\mathcal{T}_{\alpha}(t) x\right\| \leq M\|x\|, \quad\left\|\mathcal{S}_{\alpha}(t) x\right\| \leq \frac{M}{\Gamma(\alpha)}\|x\|
$$

(ii) For every $x \in \mathbb{H}, t \rightarrow \mathcal{T}_{\alpha}(t) x$ and $t \rightarrow \mathcal{S}_{\alpha}(t) x$ are continuous functions from $[0, \infty)$ into $\mathbb{H}$.

(iii) The operators $\mathcal{T}_{\alpha}(t)(t \geq 0)$ and $\mathcal{S}_{\alpha}(t)(t \geq 0)$ are strongly continuous.

(iv) If the semigroup $T(t)$ is compact, then $\mathcal{T}_{\alpha}(t)$ and $\mathcal{S}_{\alpha}(t)$ are also compact operators in $\mathbb{H}$ for $t>0$, and hence they are norm-continuous.

In this paper, we adopt the following definition of the mild solution of (1.1).

Definition 2.7 For any given $u \in L_{\mathcal{F}}^{2}([0, b], \mathbb{U})$, a stochastic process $x$ is said to be a mild solution of $(1.1)$ on $[0, b]$ if $x \in \mathcal{H}\left([0, b], L^{2}(\Omega, \mathbb{H})\right)$ and 
(i) $x(t)$ is measurable and adapted to $\mathcal{F}_{t}$;

(ii) $x(t)$ satisfies the following integral equation:

$$
\begin{aligned}
x(t)= & \mathcal{T}_{\alpha}(t) g(x)+\int_{0}^{t}(t-s)^{\alpha-1} \mathcal{S}_{\alpha}(t-s)[f(s, x(s))+B u(s)] d s \\
& +\int_{0}^{t}(t-s)^{\alpha-1} \mathcal{S}_{\alpha}(t-s) \sigma(s, x(s)) d W(s) .
\end{aligned}
$$

Let $E$ be a finite-dimensional subspace of $L^{2}(\Omega, \mathbb{H})$ and denote by $\pi_{E}$ the orthogonal projection from $L^{2}(\Omega, \mathbb{H})$ onto $E$. Let $x(b ; u)$ be the state value of system (1.1) at terminal time $b$ corresponding to control $u$. The set $\mathcal{R}(b)=\left\{x(b ; u): u \in L_{\mathcal{F}}^{2}(J, \mathbb{U})\right\}$ is called the reachable set of (1.1) at the terminal time $b$.

\section{Definition 2.8}

(a) The fractional stochastic control system (1.1) is called approximately controllable on the interval $[0, b]$ if $\overline{\mathcal{R}(b)}=L^{2}(\Omega, \mathbb{H})$.

(b) For $x_{b} \in L^{2}(\Omega, \mathbb{H})$ and $\varepsilon>0$, the fractional stochastic control system (1.1) is called finitely-approximately controllable on the interval $[0, b]$ if there exists a control $u_{\varepsilon} \in L_{\mathcal{F}}^{2}(J, \mathbb{U})$ such that the corresponding solution $x\left(b ; u_{\varepsilon}\right)$ of (1.1), satisfies the conditions

$$
\begin{aligned}
& \mathbb{E}\left\|x\left(b ; u_{\varepsilon}\right)-x_{b}\right\|^{2}<\varepsilon, \\
& \pi_{E} x\left(b ; u_{\varepsilon}\right)=\pi_{E} x_{b} .
\end{aligned}
$$

This means that the control $u_{\varepsilon}$ can be chosen such that $x\left(b ; u_{\varepsilon}\right)$ satisfies $(2.4)$ and simultaneously a finite number of constraints, that is, condition (2.5).

To prove the main result, we need the following restrictions:

(H1) The function $f:[0, b] \times \mathbb{H} \rightarrow \mathbb{H}$ is Carathéodory continuous, in addition, there exist a function $\xi_{f} \in L\left([0, b], \mathbb{R}^{+}\right)$and a nondecreasing continuous function $\Psi_{f}: \mathbb{R}^{+} \rightarrow \mathbb{R}^{+}$such that

$$
\mathbb{E}\|f(t, x)\|^{2} \leq \xi_{f}(t) \Psi_{f}\left(\mathbb{E}\|x\|^{2}\right), \quad \text { a.e. } t \in[0, b], \forall x \in \mathbb{H} \text {. }
$$

(H2) The function $\sigma:[0, b] \times \mathbb{H} \rightarrow L_{2}^{0}$ is Carathéodory continuous, in addition, there exist a function $\xi_{\sigma} \in L^{\frac{1}{q}}\left([0, b], \mathbb{R}^{+}\right)$for constant $q \in(0,2 \alpha-1)$ and a nondecreasing continuous function $\Psi_{\sigma}: \mathbb{R}^{+} \rightarrow \mathbb{R}^{+}$such that

$$
\mathbb{E}\|\sigma(t, x)\|_{L_{2}^{0}}^{2} \leq \xi_{\sigma}(t) \Psi_{\sigma}\left(\mathbb{E}\|x\|^{2}\right) \text {, a.e. } t \in[0, b], \forall x \in \mathbb{H} \text {. }
$$

(H3) $g: \mathcal{H} \rightarrow \mathbb{H}$ is continuous and $h:[0, b] \times \mathbb{H} \rightarrow \mathbb{H}$ is Carathéodory continuous, in addition, there exist function $\xi_{h} \in L\left([0, b], \mathbb{R}^{+}\right)$and a nondecreasing continuous function $\Psi_{h}: \mathbb{R}^{+} \rightarrow \mathbb{R}^{+}$such that

$$
\mathbb{E}\|h(t, x)\|^{2} \leq \xi_{h}(t) \Psi_{h}\left(\mathbb{E}\|x\|^{2}\right) \text {, a.e. } t \in[0, b], \forall x \in \mathbb{H} .
$$


(H4) The linear fractional differential system

$$
x(t)=\mathcal{T}_{\alpha}(t) x_{0}+\int_{0}^{t}(t-s)^{\alpha-1} \mathcal{S}_{\alpha}(t-s) B u(s) d s
$$

is approximately controllable in $[0, b]$.

It is known that system (2.6) approximately controllable on $[0, b]$ if and only if the condition $B^{*} \mathcal{S}_{\alpha}^{*}(b-s) \phi=0,0 \leq s \leq b$ implies that $\phi=0$.

For any $\varepsilon>0, \phi \in \mathbb{H}$ and $x \in \mathcal{H}\left([0, b], L^{2}(\Omega, \mathbb{H})\right)$, we define an important functional

$$
\begin{aligned}
J_{\varepsilon}(\phi, x)= & \frac{1}{2} \int_{0}^{b}(b-s)^{\alpha-1} \mathbb{E}\left\|B^{*} \mathcal{S}_{\alpha}^{*}(b-s) \phi\right\|^{2} d s \\
& +\varepsilon\left(\mathbb{E}\left\|\left(I-\pi_{E}\right) \phi\right\|^{2}\right)^{\frac{1}{2}}-\mathbb{E}|\phi, p(x)\rangle,
\end{aligned}
$$

where

$$
\begin{aligned}
p(x)= & x_{b}-\left(\mathcal{T}_{\alpha}(b) g(x)+\int_{0}^{b}(b-s)^{\alpha-1} \mathcal{S}_{\alpha}(b-s) f(s, x(s)) d s\right. \\
& \left.+\int_{0}^{b}(b-s)^{\alpha-1} \mathcal{S}_{\alpha}(b-s) \sigma(s, x(s)) d W(s)\right) .
\end{aligned}
$$

Lemma 2.9 Suppose that Assumptions (H1)-(H3) are satisfied. Then the following conditions hold:

(i) $p$ is continuous in $B_{r}$;

(ii) $\left\{p(x): x \in B_{r}\right\}$ is relatively compact in $\mathbb{H}$.

Proof Let $x_{n} \rightarrow x$ in $B_{r}$, then we have

$$
f\left(t, x_{n}(t)\right) \rightarrow f(t, x(t)), \quad \sigma\left(t, x_{n}(t)\right) \rightarrow \sigma(t, x(t)), \quad g\left(x_{n}\right) \rightarrow g(x) \quad(n \rightarrow \infty) .
$$

Moreover, for any $t \in[0, b]$, using Hölder inequality and Lebesgue dominated convergence theorem, we can get

$$
\begin{aligned}
& \mathbb{E}\left\|\int_{0}^{b}(b-s)^{\alpha-1} \mathcal{S}_{\alpha}(b-s)\left[f\left(s, x_{n}(s)\right)-f(s, x(s))\right] d s\right\|^{2} \\
& \quad \leq\left(\frac{M}{\Gamma(\alpha)}\right)^{2} \int_{0}^{b}(b-s)^{2 \alpha-2} d s \int_{0}^{b} \mathbb{E}\left\|f\left(s, x_{n}(s)\right)-f(s, x(s))\right\|^{2} d s \\
& \quad \leq \frac{b^{2 \alpha-1}}{2 \alpha-1}\left(\frac{M}{\Gamma(\alpha)}\right)^{2} \int_{0}^{b} \mathbb{E}\left\|f\left(s, x_{n}(s)\right)-f(s, x(s))\right\|^{2} d s \\
& \quad \rightarrow 0 \quad(n \rightarrow \infty) .
\end{aligned}
$$


On the other hand, from Lemma 2.1, Hölder inequality and Lebesgue dominated convergence theorem, we obtain

$$
\begin{aligned}
& \mathbb{E}\left\|\int_{0}^{b}(b-s)^{\alpha-1} \mathcal{S}_{\alpha}(b-s)\left[\sigma\left(s, x_{n}(s)\right)-\sigma(s, x(s))\right] d W(s)\right\|^{2} \\
& \quad \leq \operatorname{Tr}(Q)\left(\frac{M}{\Gamma(\alpha)}\right)^{2} \int_{0}^{b}(b-s)^{2 \alpha-2} \mathbb{E}\left\|\sigma\left(s, x_{n}(s)\right)-\sigma(s, x(s))\right\|^{2} d s \\
& \quad \rightarrow 0 \quad(n \rightarrow \infty) .
\end{aligned}
$$

Meanwhile, by (H3), we see that

$$
\mathbb{E}\left\|\mathcal{T}_{\alpha}(b)\left(g\left(x_{n}\right)-g(x)\right)\right\|^{2} \leq M^{2} \mathbb{E}\left\|g\left(x_{n}\right)-g(x)\right\|^{2} \rightarrow 0 \quad(n \rightarrow \infty)
$$

According to the inequality obtained above, we obtain the following relation:

$$
\begin{aligned}
\mathbb{E}\left\|p\left(x_{n}\right)-p(x)\right\|^{2} \leq & 3 \mathbb{E}\left\|\mathcal{T}_{\alpha}(b)\left(g\left(x_{n}\right)-g(x)\right)\right\|^{2} \\
& +3 \mathbb{E}\left\|\int_{0}^{b}(b-s)^{\alpha-1} \mathcal{S}_{\alpha}(b-s)\left[f\left(s, x_{n}(s)\right)-f(s, x(s))\right] d s\right\|^{2} \\
& +3 \mathbb{E}\left\|\int_{0}^{b}(b-s)^{\alpha-1} \mathcal{S}_{\alpha}(b-s)\left[\sigma\left(s, x_{n}(s)\right)-\sigma(s, x(s))\right] d W(s)\right\|^{2} \\
\rightarrow & 0 \quad(n \rightarrow \infty) .
\end{aligned}
$$

Therefore, $p$ is continuous in $B_{r}$.

Next, we prove (ii). For all $\epsilon \in(0, b)$ and all $v>0$, define an operator $F^{\epsilon, v}$ on $B_{r}$ by the formula

$$
\begin{aligned}
& \left(F^{\epsilon, v} x\right)(b) \\
& =\alpha \int_{0}^{b-\epsilon} \int_{v}^{\infty} \theta \zeta_{\alpha}(\theta)(b-s)^{\alpha-1} T\left((b-s)^{\alpha} \theta\right) f(s, x(s)) d \theta d s \\
& \quad+\alpha \int_{0}^{b-\epsilon} \int_{v}^{\infty} \theta \zeta_{\alpha}(\theta)(t-s)^{\alpha-1} T\left((b-s)^{\alpha} \theta\right) \sigma(s, x(s)) d \theta d W(s) \\
& =T\left(\epsilon^{\alpha} v\right) \alpha \int_{0}^{b-\epsilon} \int_{v}^{\infty} \theta \zeta_{\alpha}(\theta)(b-s)^{\alpha-1} T\left((b-s)^{\alpha} \theta-\epsilon^{\alpha} v\right) f(s, x(s)) d \theta d s \\
& \quad+T\left(\epsilon^{\alpha} v\right) \alpha \int_{0}^{b-\epsilon} \int_{\nu}^{\infty} \theta \zeta_{\alpha}(\theta)(b-s)^{\alpha-1} T\left((b-s)^{\alpha} \theta-\epsilon^{\alpha} v\right) \sigma(s, x(s)) d \theta d W(s) .
\end{aligned}
$$

Then the set $\left\{\left(F^{\epsilon, v} x\right)(b): x \in B_{r}\right\}$ is relatively compact in $\mathbb{H}$ because $T\left(\epsilon^{\alpha} v\right)$ is compact. We denote

$$
\begin{aligned}
\left(F_{1} x\right)(b)= & \alpha \int_{0}^{b} \int_{0}^{\infty} \theta \zeta_{\alpha}(\theta)(b-s)^{\alpha-1} T\left((b-s)^{\alpha} \theta\right) f(s, x(s)) d \theta d s \\
& +\alpha \int_{0}^{b} \int_{0}^{\infty} \theta \zeta_{\alpha}(\theta)(b-s)^{\alpha-1} T\left((b-s)^{\alpha} \theta\right) \sigma(s, x(s)) d \theta d W(s)
\end{aligned}
$$


for any $x \in B_{r}$. Applying (H1)-(H3), Lemmas 2.1 and 2.6, and Hölder inequality, we have

$$
\begin{aligned}
& \mathbb{E}\left\|\left(F_{1} x\right)(b)-\left(F^{\epsilon, v} x\right)(b)\right\|^{2} \\
& \leq 4 \mathbb{E}\left\|\alpha \int_{0}^{b} \int_{0}^{v} \theta \zeta_{\alpha}(\theta)(b-s)^{\alpha-1} T\left((b-s)^{\alpha} \theta\right) f(s, x(s)) d \theta d s\right\|^{2} \\
& +4 \mathbb{E}\left\|\alpha \int_{b-\epsilon}^{t} \int_{v}^{\infty} \theta \zeta_{\alpha}(\theta)(b-s)^{\alpha-1} T\left((t-s)^{\alpha} \theta\right) f(s, x(s)) d \theta d s\right\|^{2} \\
& +4 \mathbb{E}\left\|\alpha \int_{0}^{b} \int_{0}^{\nu} \theta \zeta_{\alpha}(\theta)(b-s)^{\alpha-1} T\left((b-s)^{\alpha} \theta\right) \sigma(s, x(s)) d \theta d W(s)\right\|^{2} \\
& +4 \mathbb{E}\left\|\alpha \int_{b-\epsilon}^{b} \int_{v}^{\infty} \theta \zeta_{\alpha}(\theta)(b-s)^{\alpha-1} T\left((b-s)^{\alpha} \theta\right) \sigma(s, x(s)) d \theta d W(s)\right\|^{2} \\
& \leq 4 M^{2} \alpha^{2} \int_{0}^{b}(b-s)^{2 \alpha-2} d s \int_{0}^{b} \mathbb{E}\|f(s, x(s))\|^{2} d s\left(\int_{0}^{\nu} \theta \zeta_{\alpha}(\theta) d \theta\right)^{2} \\
& +\frac{4 M^{2} \alpha^{2}}{\Gamma^{2}(1+\alpha)} \int_{b-\epsilon}^{b}(b-s)^{2 \alpha-2} d s \int_{b-\epsilon}^{b} \mathbb{E}\|f(s, x(s))\|^{2} d s \\
& +4 \operatorname{Tr}(Q) M^{2} \alpha^{2} \int_{0}^{b}(b-s)^{2 \alpha-2} \mathbb{E}\|\sigma(s, x(s))\|_{L_{2}^{0}}^{2} d s\left(\int_{0}^{\nu} \theta \zeta_{\alpha}(\theta) d \theta\right)^{2} \\
& +\frac{4 \operatorname{Tr}(Q) M^{2} \alpha^{2}}{\Gamma^{2}(1+\alpha)} \int_{t-\epsilon}^{b}(b-s)^{2 \alpha-2} \mathbb{E}\|\sigma(s, x(s))\|_{L_{2}^{0}}^{2} d s \\
& \leq \frac{4 M^{2} \alpha^{2} b^{2 \alpha-1} \Psi_{f}(R)\left\|\xi_{f}\right\|_{L[0, b]}}{2 \alpha-1}\left(\int_{0}^{v} \theta \zeta_{\alpha}(\theta) d \theta\right)^{2} \\
& +\frac{4 M^{2} \alpha^{2} \Psi_{f}(R)\left\|\xi_{f}\right\|_{L[0, b]}}{\Gamma^{2}(1+\alpha)} \frac{\epsilon^{2 \alpha-1}}{2 \alpha-1} \\
& +4 \operatorname{Tr}(Q) M^{2} \alpha^{2} \Psi_{\sigma}(R)\left(\frac{1-q}{2 \alpha-1-q}\right)^{1-q}\left\|\xi_{\sigma}\right\|_{L^{\frac{1}{q}}}\left(\int_{0}^{v} \theta \zeta_{\alpha}(\theta) d \theta\right)^{2} \\
& +\frac{4 \operatorname{Tr}(Q) M^{2} \alpha^{2} \Psi_{\sigma}(R)}{\Gamma^{2}(1+\alpha)}\left(\frac{1-q}{2 \alpha-1-q}\right)^{1-q}\left\|\xi_{\sigma}\right\|_{L^{\frac{1}{q}}} \epsilon^{2 \alpha-1-q} \\
& \rightarrow 0 \quad(\epsilon, \nu \rightarrow 0) .
\end{aligned}
$$

Therefore, there are relatively compact sets arbitrarily close to the set $\left\{\left(F_{1} x\right)(b): x \in B_{r}\right\}$ in $\mathbb{H}$. Hence the set $\left\{\left(F_{1} x\right)(b): x \in B_{r}\right\}$ is relatively compact in $\mathbb{H}$. Combining this fact with the compactness of $\mathcal{T}_{\alpha}(b)$, it follows that $\left\{p(x): x \in B_{r}\right\}$ is relatively compact in $\mathbb{H}$.

This completes the proof of Lemma 2.9.

Lemma 2.10 Suppose that Assumptions $(\mathrm{H} 1)-(\mathrm{H} 4)$ are satisfied. Then the functional $J_{\varepsilon}$ satisfies the following properties:

(i) For $x \in \mathcal{H}\left([0, b], L^{2}(\Omega, \mathbb{H})\right)$, the map $\phi \rightarrow J_{\varepsilon}(\phi, x)$ is continuous and strictly convex;

(ii) For any $r>0$,

$$
\lim _{\left(\mathbb{E}\|\phi\|^{2}\right)^{\frac{1}{2}} \rightarrow \infty} \inf _{x \in B_{r}} \frac{J_{\varepsilon}(\phi, x)}{\left(\mathbb{E}\|\phi\|^{2}\right)^{\frac{1}{2}}} \geq \varepsilon .
$$


Proof (i) From the definition of $J_{\varepsilon}(\phi, x)$, it is easy to see that $\phi \rightarrow J_{\varepsilon}(\phi, x)$ is continuous and strictly convex.

(ii) If (ii) does not hold, there exist sequences $\left\{x_{n}\right\} \subset \mathcal{H}\left([0, b], L^{2}(\Omega, \mathbb{H})\right)$ and $\left\{\phi_{n}\right\} \subset \mathbb{H}$ with $\left(\mathbb{E}\left\|\phi_{n}\right\|^{2}\right)^{\frac{1}{2}} \rightarrow \infty$ such that

$$
\varliminf_{n \rightarrow \infty} \frac{J_{\varepsilon}\left(\phi_{n}, x_{n}\right)}{\left(\mathbb{E}\left\|\phi_{n}\right\|^{2}\right)^{\frac{1}{2}}}<\varepsilon
$$

By Lemma 2.9, the set $\left\{p\left(x_{n}\right): x_{n} \in B_{r}\right\}$ is relatively compact in $\mathbb{H}$. Hence there is a subsequence, still denoted by $p\left(x_{n}\right)$, that strongly converges to, say, $p_{0}$ in $\mathbb{H}$.

We normalize $\phi_{n}$ as $\widetilde{\phi}_{n}=\frac{\phi_{n}}{\left(\mathbb{E}\left\|\phi_{n}\right\|^{2}\right)^{\frac{1}{2}}}$. Evidently, $\left\|\widetilde{\phi}_{n}\right\|_{L^{2}}=1$, so we can extract a subsequence (still denoted by $\widetilde{\phi}_{n}$ ), which weakly converges to an element $\widetilde{\phi}$ in $\mathbb{H}$. By the compactness of $T(t)$, we infer that $B^{*} \mathcal{S}_{\alpha}^{*}(b-s) \widetilde{\phi}_{n}$ strongly converges to $B^{*} \mathcal{S}_{\alpha}^{*}(b-s) \widetilde{\phi}$. By $(2.7)$, we obtain

$$
\begin{aligned}
\frac{J_{\varepsilon}\left(\phi_{n}, x_{n}\right)}{\left(\mathbb{E}\left\|\phi_{n}\right\|^{2}\right)^{\frac{1}{2}}}= & \frac{\left(\mathbb{E}\left\|\phi_{n}\right\|^{2}\right)^{\frac{1}{2}}}{2} \int_{0}^{b}(b-s)^{\alpha-1} \mathbb{E}\left\|B^{*} \mathcal{S}_{\alpha}^{*}(b-s) \widetilde{\phi}_{n}\right\|^{2} d s \\
& +\varepsilon\left(\mathbb{E}\left\|\left(I-\pi_{E}\right) \widetilde{\phi}_{n}\right\|^{2}\right)^{\frac{1}{2}}-\mathbb{E}\left\langle\widetilde{\phi}_{n}, p\left(x_{n}\right)\right\rangle,
\end{aligned}
$$

Observe that (2.12) and Fatou lemma implies that

$$
\begin{aligned}
& \int_{0}^{b}(b-s)^{\alpha-1} \mathbb{E}\left\|B^{*} \mathcal{S}_{\alpha}^{*}(b-s) \widetilde{\phi}\right\|^{2} d s \\
& \quad \leq \varliminf_{n \rightarrow \infty} \int_{0}^{b}(b-s)^{\alpha-1} \mathbb{E}\left\|B^{*} \mathcal{S}_{\alpha}^{*}(b-s) \widetilde{\phi}_{n}\right\|^{2} d s=0 .
\end{aligned}
$$

By (H4), we get $\widetilde{\phi}=0$, that is, $\widetilde{\phi}_{n}$ weakly converges to 0 in $\mathbb{H}$. As $E$ is finite-dimensional, the orthogonal projection $\pi_{E}$ is compact. Moreover, we have

$$
\lim _{n \rightarrow \infty}\left(\mathbb{E}\left\|\left(I-\pi_{E}\right) \widetilde{\phi}_{n}\right\|^{2}\right)^{\frac{1}{2}}=1 \text {. }
$$

Therefore,

$$
\varepsilon>\lim _{n \rightarrow \infty} \frac{J_{\varepsilon}\left(\phi_{n}, x_{n}\right)}{\left(\mathbb{E}\left\|\phi_{n}\right\|^{2}\right)^{\frac{1}{2}}} \geq \varliminf_{n \rightarrow \infty}\left[\varepsilon\left(\mathbb{E}\left\|\left(I-\pi_{E}\right) \widetilde{\phi}_{n}\right\|^{2}\right)^{\frac{1}{2}}-\mathbb{E}\left\langle\widetilde{\phi}_{n}, p\left(x_{n}\right)\right\rangle\right]=\varepsilon,
$$

which contradicts (2.12). So (ii) holds.

This completes the proof of Lemma 2.10 .

Inequality (2.11) means that the functional $J_{\varepsilon}(\cdot, x): \mathbb{H} \rightarrow \mathbb{R}$ is coercive. Thus, for any $x \in \mathcal{H}\left([0, b], L^{2}(\Omega, \mathbb{H})\right)$, the functional $J_{\varepsilon}(\cdot, x)$ admits a unique critical point $\widehat{\phi}_{\varepsilon}$. We define a mapping $\Phi_{\varepsilon}: \mathcal{H}\left([0, b], L^{2}(\Omega, \mathbb{H})\right) \rightarrow \mathbb{H}$ by $\Phi_{\varepsilon}(x)=\widehat{\phi}_{\varepsilon}$. Next, we estimate some properties of mapping $\Phi_{\varepsilon}$.

Lemma 2.11 If Assumptions (H1)-(H4) are satisfied, then the following conclusions hold:

(i) For any $x \in B_{r}$, there exists a constant $L_{\varepsilon}>0$ such that $\mathbb{E}\left\|\Phi_{\varepsilon}(x)\right\|^{2} \leq L_{\varepsilon}$; 
(ii) For any $x_{n}, x \in B_{r}$ satisfying $x_{n} \rightarrow x$ in $\mathcal{H}\left([0, b], L^{2}(\Omega, \mathbb{H})\right)$, it holds that

$$
\lim _{n \rightarrow \infty} \mathbb{E}\left\|\Phi_{\varepsilon}\left(x_{n}\right)-\Phi_{\varepsilon}(x)\right\|^{2}=0
$$

Proof (i) By Lemma 2.10, there exists $L_{\varepsilon}>0$ such that

$$
\inf _{x \in B_{r}} \frac{J_{\varepsilon}(\phi, x)}{\left(\mathbb{E}\|\phi\|^{2}\right)^{\frac{1}{2}}} \geq \frac{\varepsilon}{2}, \quad \mathbb{E}\|\phi\|^{2}>L_{\varepsilon} .
$$

On the other hand, by the definition of $\Phi_{\varepsilon}$,

$$
J_{\varepsilon}\left(\Phi_{\varepsilon}(x), x\right) \leq J_{\varepsilon}(0, x)=0, \quad x \in B_{r}
$$

Therefore, by (2.13) and (2.14), we obtain that

$$
\mathbb{E}\left\|\Phi_{\varepsilon}(x)\right\|^{2} \leq L_{\varepsilon}, \quad x \in B_{r}
$$

(ii) Set $\widehat{\phi}_{\varepsilon, n}=\Phi_{\varepsilon}\left(x_{n}\right)$ and $\widehat{\phi}_{\varepsilon}=\Phi_{\varepsilon}(x)$. By (i), we know that $\left\{\widehat{\phi}_{\varepsilon, n}\right\}$ is bounded, thus we suppose that $\widehat{\phi}_{\varepsilon, n}$ weakly converges to $\widetilde{\phi}_{\varepsilon}$ in $\mathbb{H}$. According to the definition of $J_{\varepsilon}$, Fatou lemma and the optimality of both $\widehat{\phi}_{\varepsilon, n}=\Phi_{\varepsilon}\left(x_{n}\right)$ and $\widehat{\phi}_{\varepsilon}=\Phi_{\varepsilon}(x)$, we have

$$
\begin{aligned}
& J_{\varepsilon}\left(\widehat{\phi}_{\varepsilon}, x\right) \leq J_{\varepsilon}\left(\widetilde{\phi}_{\varepsilon}, x\right) \leq \underline{\lim }_{n \rightarrow \infty} J_{\varepsilon}\left(\widehat{\phi}_{\varepsilon, n}, x_{n}\right) \leq \varlimsup_{n \rightarrow \infty} J_{\varepsilon}\left(\widehat{\phi}_{\varepsilon, n}, x_{n}\right) \\
& \leq \lim _{n \rightarrow \infty} J_{\varepsilon}\left(\widehat{\phi}_{\varepsilon}, x_{n}\right)=J_{\varepsilon}\left(\widehat{\phi}_{\varepsilon}, x\right) \text {. }
\end{aligned}
$$

Thus, $J_{\varepsilon}\left(\widehat{\phi}_{\varepsilon}, x\right)=J_{\varepsilon}\left(\widetilde{\phi}_{\varepsilon}, x\right)$, that is, $\widetilde{\phi}_{\varepsilon}$ is also a minimum of $J_{\varepsilon}(\cdot, x)$. By the uniqueness of the minimum of $J_{\varepsilon}(\cdot, x)$, we get $\widehat{\phi}_{\varepsilon}=\widetilde{\phi}_{\varepsilon}$. Moreover, we have

$$
\begin{aligned}
& \lim _{n \rightarrow \infty} J_{\varepsilon}\left(\widehat{\phi}_{\varepsilon, n}, x_{n}\right)=J_{\varepsilon}\left(\widehat{\phi}_{\varepsilon}, x\right), \\
& \lim _{n \rightarrow \infty} \int_{0}^{b}(b-s)^{\alpha-1} \mathbb{E}\left\|B^{*} \mathcal{S}_{\alpha}^{*}(b-s) \widehat{\phi}_{\varepsilon, n}\right\|^{2} d s \\
& \quad=\int_{0}^{b}(b-s)^{\alpha-1} \mathbb{E}\left\|B^{*} \mathcal{S}_{\alpha}^{*}(b-s) \widehat{\phi}_{\varepsilon}\right\|^{2} d s, \\
& \lim _{n \rightarrow \infty} \mathbb{E}\left(\widehat{\phi}_{\varepsilon, n}, p\left(x_{n}\right)\right\rangle=\mathbb{E}\left\langle\widehat{\phi}_{\varepsilon}, p(x)\right\rangle, \\
& \left(\mathbb{E}\left\|\left(I-\pi_{E}\right) \widehat{\phi}_{\varepsilon}\right\|^{2}\right)^{\frac{1}{2}} \leq \underline{\lim }\left(\mathbb{E}\left\|\left(I-\pi_{E}\right) \widehat{\phi}_{\varepsilon, n}\right\|^{2}\right)^{\frac{1}{2}} .
\end{aligned}
$$

From these relations, we easily see that

$$
\lim _{n \rightarrow \infty}\left(\mathbb{E}\left\|\left(I-\pi_{E}\right) \widehat{\phi}_{\varepsilon, n}\right\|^{2}\right)^{\frac{1}{2}}=\left(\mathbb{E}\left\|\left(I-\pi_{E}\right) \widehat{\phi}_{\varepsilon}\right\|^{2}\right)^{\frac{1}{2}}
$$

Since $\mathbb{H}$ is a Hilbert space, by $(2.16)$ and since $\widehat{\phi}_{\varepsilon, n} \rightarrow \widehat{\phi}_{\varepsilon}$ weakly in $\mathbb{H}$, we have

$$
\lim _{n \rightarrow \infty} \mathbb{E}\left\|\Phi_{\varepsilon}\left(x_{n}\right)-\Phi_{\varepsilon}(x)\right\|^{2}=0
$$

This completes the proof of Lemma 2.11. 
Now, we introduce a control $u_{\varepsilon}(t, x)$ by

$$
u_{\varepsilon}(t, x)=B^{*} \mathcal{S}_{\alpha}^{*}(b-t) \Phi_{\varepsilon}(x)
$$

From Lemma 2.11, we can get the following obvious result:

Lemma 2.12 If Assumptions (H1)-(H4) are satisfied, then for any $x \in B_{r}$, the following conclusions hold:

(i) $\mathbb{E}\left\|u_{\varepsilon}(t, x)\right\|^{2} \leq L_{u}$;

(ii) $u_{\varepsilon}(t, x)$ is continuous in $B_{r}$, where $L_{u}=\|B\|^{2}\left(\frac{M}{\Gamma(\alpha)}\right)^{2} L_{\varepsilon}$.

To discuss the finite-controllability for system (1.1), we need the following lemmas in this paper.

Lemma 2.13 Assume that-A generates a compact $C_{0}$-semigroup $T(t)(t \geq 0)$ of uniformly bounded operators in a Hilbert space $\mathbb{H}$. Let Assumptions (H1)-(H4) hold. Suppose, in addition, that the following condition is satisfied:

(H5) There is a constant $\delta \in(0, b)$ such that for any $t \in[0, b]$,

$$
f\left(t, x_{1}(t)\right)=f\left(t, x_{2}(t)\right), \quad \sigma\left(t, x_{1}(t)\right)=\sigma\left(t, x_{2}(t)\right), \quad h\left(t, x_{1}(t)\right)=h\left(t, x_{2}(t)\right),
$$

where $x_{1}, x_{2} \in \mathcal{H}\left([0, b], L^{2}(\Omega, \mathbb{H})\right)$ with $x_{1}(t)=x_{2}(t)(t \in[\delta, b])$.

Then the nonlocal problem (1.1) has at least one mild solution in $B_{R}$ provided that there exists a positive constant $R$ such that

$$
\begin{aligned}
& 3 M^{2} b \Psi_{h}(R)\left\|\xi_{h}\right\|_{L[0, b]}+3 c_{0}\left(2 \Psi_{f}(R)\left\|\xi_{f}\right\|_{L[0, b]}+2 b\|B\|^{2} L_{u}\right) \\
& \quad+3 c_{1} \operatorname{Tr}(Q) \Psi_{\sigma}(R)\left\|\xi_{\sigma}\right\|_{L^{\frac{1}{q}}} \leq R .
\end{aligned}
$$

where

$$
c_{0}=\left(\frac{M}{\Gamma(\alpha)}\right)^{2} \frac{b^{2 \alpha-1}}{2 \alpha-1}, \quad c_{1}=\left(\frac{M}{\Gamma(\alpha)}\right)^{2}\left(\frac{1-q}{2 \alpha-1-q}\right)^{1-q} b^{2 \alpha-1-q}
$$

Proof For any $r>0$, define

$$
B_{r}(\delta)=\left\{x \in \mathcal{H}\left([\delta, b], L^{2}(\Omega, \mathbb{H})\right): \mathbb{E}\|x(t)\|^{2} \leq r, \forall t \in[\delta, b]\right\}
$$

It is easily seen that for each $x \in B_{r}(\delta)$, there exists a function $y \in B_{r}$ satisfying $x(t)=y(t)$, $t \in[\delta, b]$. Define the following mappings on $B_{r}(\delta)$ by

$$
\begin{aligned}
& \left(f^{*} x\right)(t)=f(t, y(t)), \quad t \in[0, b], \\
& \left(\sigma^{*} x\right)(t)=\sigma(t, y(t)), \quad t \in[0, b], \\
& g^{*}(x)=g(y) .
\end{aligned}
$$


Then, by conditions $(\mathrm{H} 1)-(\mathrm{H} 3)$ and $(\mathrm{H} 5)$, it is easy to see that $f^{*}, \sigma^{*}, g^{*}$ is well defined on $B_{r}(\delta)$ and continuous. In addition,

$$
\begin{aligned}
& \mathbb{E}\left\|\left(f^{*} x\right)(t)\right\|^{2} \leq \xi_{f}(t) \Psi_{f}\left(\mathbb{E}\|x\|^{2}\right), \quad \text { a.e. } t \in[0, b], \forall x \in B_{r}(\delta), \\
& \mathbb{E}\left\|\left(\sigma^{*} x\right)(t)\right\|_{L_{2}^{0}}^{2} \leq \xi_{\sigma}(t) \Psi_{\sigma}\left(\mathbb{E}\|x\|^{2}\right), \quad \text { a.e. } t \in[0, b], \forall x \in B_{r}(\delta), \\
& \mathbb{E}\left\|g^{*}(x)\right\|^{2} \leq b \Psi_{h}(r)\left\|\xi_{h}\right\|_{L[0, b]}, \quad \forall x \in B_{r}(\delta) .
\end{aligned}
$$

Define an operator $F_{\delta}$ on $B_{r}(\delta)$ as follows:

$$
\begin{aligned}
\left(F_{\delta} x\right)(t)= & \mathcal{T}_{\alpha}(t) g^{*}(x)+\int_{0}^{t}(t-s)^{\alpha-1} \mathcal{S}_{\alpha}(t-s)\left[\left(f^{*} x\right)(s)+B u_{\varepsilon}^{*}(s, x)\right] d s \\
& +\int_{0}^{t}(t-s)^{\alpha-1} \mathcal{S}_{\alpha}(t-s)\left(\sigma^{*} x\right)(s) d W(s), \quad t \in[\delta, b],
\end{aligned}
$$

here $u_{\varepsilon}^{*}(s, x)$ is defined by $u_{\varepsilon}^{*}(t, x)=B^{*} \mathcal{S}_{\alpha}^{*}(b-t) \Phi_{\varepsilon}^{*}(x)$, and $\Phi_{\varepsilon}^{*}(x)$ is the critical point of $J_{\varepsilon}(\phi, x)$ of $p^{*}(x)$, where

$$
\begin{aligned}
p^{*}(x)= & x_{b}-\left(\mathcal{T}_{\alpha}(b) g^{*}(x)+\int_{0}^{b}(b-s)^{\alpha-1} \mathcal{S}_{\alpha}(b-s)\left(f^{*} x\right)(s) d s\right. \\
& \left.+\int_{0}^{b}(b-s)^{\alpha-1} \mathcal{S}_{\alpha}(b-s)\left(\sigma^{*} x\right)(s) d W(s)\right) .
\end{aligned}
$$

Evidently, the results in Lemma 2.12 hold for $u_{\varepsilon}^{*}(s, x)$.

Next we prove that $F_{\delta}$ has a fixed point by Schauder's fixed point theorem. For this purpose, we first check that there is a positive number $R$ such that $F_{\delta}$ maps $B_{R}(\delta)$ into itself. For any $x \in B_{R}(\delta)$ and $t \in[\delta, b]$, it follows from (2.17), (2.18), Lemmas 2.1 and 2.6, and Hölder inequality that

$$
\begin{aligned}
\mathbb{E} \|( & \left.F_{\delta} x\right)(t) \|^{2} \\
\leq & 3 \mathbb{E}\left\|\mathcal{T}_{\alpha}(t) g^{*}(x)\right\|^{2}+3 \mathbb{E}\left\|\int_{0}^{t}(t-s)^{\alpha-1} \mathcal{S}_{\alpha}(t-s)\left[\left(f^{*} x\right)(s)+B u_{\varepsilon}^{*}(s, x)\right] d s\right\|^{2} \\
& +3 \mathbb{E}\left\|\int_{0}^{t_{r}}\left(t_{r}-s\right)^{\alpha-1} \mathcal{S}_{\alpha}\left(t_{r}-s\right)\left(\sigma^{*} x\right)(s) d W(s)\right\|^{2} \\
\leq & 3 M^{2} b \Psi_{h}(r)\left\|\xi_{h}\right\|_{L[0, b]}+\frac{3 M^{2}}{\Gamma^{2}(\alpha)} \cdot \frac{b^{2 \alpha-1}}{2 \alpha-1} \int_{0}^{t} \mathbb{E}\left\|\left(f^{*} x\right)(s)+B u_{\varepsilon}^{*}(s, x)\right\|^{2} d s \\
& +\frac{3 \operatorname{Tr}(Q) M^{2}}{\Gamma^{2}(\alpha)} \int_{0}^{t}(t-s)^{2 \alpha-2} \mathbb{E}\left\|\left(\sigma^{*} x\right)(s)\right\|_{L_{2}^{0}}^{2} d s \\
\leq & 3 M^{2} b \Psi_{h}(R)\left\|\xi_{h}\right\|_{L[0, b]}+3 c_{0}\left(2 \Psi_{f}(R)\left\|\xi_{f}\right\|_{L[0, b]}+2 b\|B\|^{2} L_{u}\right) \\
& +3 c_{1} \operatorname{Tr}(Q) \Psi_{\sigma}(R)\left\|\xi_{\sigma}\right\|_{L^{\frac{1}{q}}} \\
\leq & R .
\end{aligned}
$$

It then follows that $F_{\delta}$ maps $B_{R}(\delta)$ to $B_{R}(\delta)$. 
Secondly, with a method similar to that in the proof of Lemma 2.9, we can also prove that $F_{\delta}: B_{R}(\delta) \rightarrow B_{R}(\delta)$ is a continuous operator and the set $\left\{\left(F_{\delta} x\right)(t): x \in B_{R}(\delta)\right\}$ is relatively compact in $\mathbb{H}$ for $t \in[\delta, b]$.

In what follows, we will show that $F_{\delta}\left(B_{R}(\delta)\right)$ is an equicontinuous family of functions on $[\delta, b]$. For any $x \in B_{R}(\delta)$ and $\delta \leq t_{1}<t_{2} \leq b$, we get that

$$
\begin{aligned}
\mathbb{E}\left\|\left(F_{\delta} x\right)\left(t_{2}\right)-\left(F_{\delta} x\right)\left(t_{1}\right)\right\|^{2} \\
=7 \mathbb{E}\left\|\left(\mathcal{T}_{\alpha}\left(t_{2}\right)-\mathcal{T}_{\alpha}\left(t_{2}\right)\right) g^{*}(x)\right\|^{2} \\
\quad+7 \mathbb{E}\left\|\int_{t_{1}}^{t_{2}}\left(t_{2}-s\right)^{\alpha-1} \mathcal{S}_{\alpha}\left(t_{2}-s\right)\left[\left(f^{*} x\right)(s)+B u_{\varepsilon}^{*}(s, x)\right] d s\right\|^{2} \\
+7 \mathbb{E}\left\|\int_{0}^{t_{1}}\left[\left(t_{2}-s\right)^{\alpha-1}-\left(t_{1}-s\right)^{\alpha-1}\right] \mathcal{S}_{\alpha}\left(t_{2}-s\right)\left[\left(f^{*} x\right)(s)+B u_{\varepsilon}^{*}(s, x)\right]\right\|^{2} \\
\quad+7 \mathbb{E}\left\|\int_{0}^{t_{1}}\left(t_{1}-s\right)^{\alpha-1}\left[\mathcal{S}_{\alpha}\left(t_{2}-s\right)-\mathcal{S}_{\alpha}\left(t_{1}-s\right)\right]\left[\left(f^{*} x\right)(s)+B u_{\varepsilon}^{*}(s, x)\right]\right\|^{2} \\
\quad+7 \mathbb{E}\left\|\int_{t_{1}}^{t_{2}}\left(t_{2}-s\right)^{\alpha-1} \mathcal{S}_{\alpha}\left(t_{2}-s\right)\left(\sigma^{*} x\right)(s) d W(s)\right\|^{2} \\
\quad+7 \mathbb{E}\left\|\int_{0}^{t_{1}}\left[\left(t_{2}-s\right)^{\alpha-1}-\left(t_{1}-s\right)^{\alpha-1}\right] \mathcal{S}_{\alpha}\left(t_{2}-s\right)\left(\sigma^{*} x\right)(s) d W(s)\right\|^{2} \\
\quad+7 \mathbb{E}\left\|\int_{0}^{t_{1}}\left(t_{1}-s\right)^{\alpha-1}\left[\mathcal{S}_{\alpha}\left(t_{2}-s\right)-\mathcal{S}_{\alpha}\left(t_{1}-s\right)\right]\left(\sigma^{*} x\right)(s) d W(s)\right\|^{2} \\
=I_{0}+I_{1}+I_{2}+I_{3}+I_{4}+I_{5}+I_{6} .
\end{aligned}
$$

In order to prove that $\mathbb{E}\left\|\left(F_{1} x\right)\left(t_{2}\right)-\left(F_{1} x\right)\left(t_{1}\right)\right\|^{2} \rightarrow 0\left(t_{2}-t_{1} \rightarrow 0\right)$, we only need to check $I_{i} \rightarrow 0$ independently of $x \in B_{R}(\delta)$ when $t_{2}-t_{1} \rightarrow 0$ for $i=0,1,2, \ldots, 6$. Clearly, $I_{0} \rightarrow 0$ as $t_{2}-t_{1} \rightarrow 0$.

For $I_{1}$ and $I_{4}$, from (2.18), Lemmas 2.1 and 2.6, and Hölder inequality, we obtain the estimates

$$
\begin{aligned}
I_{1} & =7 \mathbb{E}\left\|\int_{t_{1}}^{t_{2}}\left(t_{2}-s\right)^{\alpha-1} \mathcal{S}_{\alpha}\left(t_{2}-s\right)\left[\left(f^{*} x\right)(s)+B u_{\varepsilon}^{*}(s, x)\right] d s\right\|^{2} \\
& \leq \frac{7 M^{2}}{\Gamma^{2}(\alpha)} \int_{t_{1}}^{t_{2}}\left(t_{2}-s\right)^{2 \alpha-2} d s \int_{t_{1}}^{t_{2}} \mathbb{E}\left\|\left(f^{*} x\right)(s)+B u_{\varepsilon}^{*}(s, x)\right\|^{2} d s \\
& \leq \frac{7 M^{2}\left(2 \Psi_{f}(R)\left\|\xi_{f}\right\|_{L[0, b]}+2\|B\|^{2} L_{u} b\right)}{\Gamma^{2}(\alpha)} \cdot \frac{\left(t_{2}-t_{1}\right)^{2 \alpha-1}}{2 \alpha-1} \\
& \rightarrow 0 \quad\left(t_{2}-t_{1} \rightarrow 0\right), \\
I_{4} & =7 \mathbb{E}\left\|\int_{t_{1}}^{t_{2}}\left(t_{2}-s\right)^{\alpha-1} \mathcal{S}_{\alpha}\left(t_{2}-s\right)\left(\sigma^{*} x\right)(s) d W(s)\right\|^{2} \\
& \leq \frac{7 \operatorname{Tr}(Q) M^{2}}{\Gamma^{2}(\alpha)} \int_{t_{1}}^{t_{2}}\left(t_{2}-s\right)^{2 \alpha-2} \mathbb{E}\left\|\left(\sigma^{*} x\right)(s)\right\|^{2} d s \\
& \leq \frac{7 \operatorname{Tr}(Q) M^{2} \Psi_{\sigma}(R)\left\|\xi_{\sigma}\right\| L_{L^{\frac{1}{\beta}}}\left(\frac{1-q}{2 \alpha-1-q}\right)^{1-q}\left(t_{2}-t_{1}\right)^{2 \alpha-1-q}}{\Gamma^{2}(\alpha)} \\
& \rightarrow 0 \quad\left(t_{2}-t_{1} \rightarrow 0\right) .
\end{aligned}
$$


Similarly, for $I_{2}$ and $I_{5}$, we get

$$
\begin{aligned}
& I_{2}=7 \mathbb{E} \| \int_{0}^{t_{1}}\left[\left(t_{2}-s\right)^{\alpha-1}-\left(t_{1}-s\right)^{\alpha-1}\right] \mathcal{S}_{\alpha}\left(t_{2}-s\right) \\
& \times\left[\left(f^{*} x\right)(s)+B u_{\varepsilon}^{*}(s, x)\right] d s \|^{2} \\
& \leq \frac{7 M^{2}}{\Gamma^{2}(\alpha)} \int_{0}^{t_{1}}\left[\left(t_{2}-s\right)^{\alpha-1}-\left(t_{1}-s\right)^{\alpha-1}\right]^{2} d s \\
& \times \int_{0}^{t_{1}} \mathbb{E}\left\|\left(f^{*} x\right)(s)+B u_{\varepsilon}^{*}(s, x)\right\|^{2} d s \\
& \leq \frac{7 M^{2}\left(2 \Psi_{f}(R)\left\|\xi_{f}\right\|_{L[0, b]}+2\|B\|^{2} L_{u} b\right)}{\Gamma^{2}(\alpha)} \\
& \times \int_{0}^{t_{1}}\left[\left(t_{2}-s\right)^{\alpha-1}-\left(t_{1}-s\right)^{\alpha-1}\right]^{2} d s \\
& \rightarrow 0 \quad\left(t_{2}-t_{1} \rightarrow 0\right), \\
& I_{5}=7 \mathbb{E}\left\|\int_{0}^{t_{1}}\left[\left(t_{2}-s\right)^{\alpha-1}-\left(t_{1}-s\right)^{\alpha-1}\right] \mathcal{S}_{\alpha}\left(t_{2}-s\right)\left(\sigma^{*} x\right)(s) d W(s)\right\|^{2} \\
& \leq \frac{7 \operatorname{Tr}(Q) M^{2}}{\Gamma^{2}(\alpha)} \int_{0}^{t_{1}}\left[\left(t_{2}-s\right)^{\alpha-1}-\left(t_{1}-s\right)^{\alpha-1}\right]^{2} \mathbb{E}\left\|\left(\sigma^{*} x\right)(s)\right\|^{2} d s \\
& \leq \frac{7 \operatorname{Tr}(Q) M^{2} \Psi_{\sigma}(R)\left\|\xi_{\sigma}\right\|_{L^{\frac{1}{q}}}}{\Gamma^{2}(\alpha)} \\
& \times\left(\int_{0}^{t_{1}}\left[\left(t_{2}-s\right)^{\alpha-1}-\left(t_{1}-s\right)^{\alpha-1}\right]^{\frac{2}{1-q}} d s\right)^{1-q} \\
& \rightarrow 0 \quad\left(t_{2}-t_{1} \rightarrow 0\right) \text {. }
\end{aligned}
$$

Further, for $I_{3}$ and $I_{6}$, suppose $0<\varepsilon<t_{1}$ is small enough, we obtain the following inequalities:

$$
\begin{aligned}
I_{3}= & 7 \mathbb{E} \| \int_{0}^{t_{1}}\left(t_{1}-s\right)^{\alpha-1}\left[\mathcal{S}_{\alpha}\left(t_{2}-s\right)-\mathcal{S}_{\alpha}\left(t_{1}-s\right)\right] \\
& \times\left[\left(f^{*} x\right)(s)+B u_{\varepsilon}^{*}(s, x)\right] d s \|^{2} \\
\leq & 14 \mathbb{E} \| \int_{0}^{t_{1}-\varepsilon}\left(t_{1}-s\right)^{\alpha-1}\left[\mathcal{S}_{\alpha}\left(t_{2}-s\right)-\mathcal{S}_{\alpha}\left(t_{1}-s\right)\right] \\
& \times\left[\left(f^{*} x\right)(s)+B u_{\varepsilon}^{*}(s, x)\right] d s \|^{2} \\
& +14 \mathbb{E} \| \int_{t_{1}-\varepsilon}^{t_{1}}\left(t_{1}-s\right)^{\alpha-1}\left[\mathcal{S}_{\alpha}\left(t_{2}-s\right)-\mathcal{S}_{\alpha}\left(t_{1}-s\right)\right] \\
& \times\left[\left(f^{*} x\right)(s)+B u_{\varepsilon}^{*}(s, x)\right] d s \|^{2} \\
\leq & 14 \sup _{s \in\left[0, t_{1}-\varepsilon\right]}\left\|\mathcal{S}_{\alpha}\left(t_{2}-s\right)-\mathcal{S}_{\alpha}\left(t_{1}-s\right)\right\|^{2}\left(2 \Psi_{f}(R)\left\|\xi_{f}\right\|_{L[0, b]}+2\|B\|^{2} L_{u} b\right)
\end{aligned}
$$




$$
\begin{aligned}
& \times \frac{t_{1}^{2 \alpha-1}-\varepsilon^{2 \alpha-1}}{2 \alpha-1} \\
& +14\left(\frac{2 M}{\Gamma(\alpha)}\right)^{2}\left(2 \Psi_{f}(R)\left\|\xi_{f}\right\|_{L[0, b]}+2\|B\|^{2} L_{u} b\right) \frac{\varepsilon^{2 \alpha-1}}{2 \alpha-1} \\
\rightarrow & 0 \quad\left(t_{2}-t_{1} \rightarrow 0 \text { and } \varepsilon \rightarrow 0\right), \\
I_{6}= & 7 \mathbb{E}\left\|\int_{0}^{t_{1}}\left(t_{1}-s\right)^{\alpha-1}\left[\mathcal{S}_{\alpha}\left(t_{2}-s\right)-\mathcal{S}_{\alpha}\left(t_{1}-s\right)\right]\left(\sigma^{*} x\right)(s)\right\|^{2} \\
\leq & 14 \mathbb{E}\left\|\int_{0}^{t_{1}-\varepsilon}\left(t_{1}-s\right)^{\alpha-1}\left[\mathcal{S}_{\alpha}\left(t_{2}-s\right)-\mathcal{S}_{\alpha}\left(t_{1}-s\right)\right]\left(\sigma^{*} x\right)(s) d s\right\|^{2} \\
& +14 \mathbb{E}\left\|\int_{t_{1}-\varepsilon}^{t_{1}}\left(t_{1}-s\right)^{\alpha-1}\left[\mathcal{S}_{\alpha}\left(t_{2}-s\right)-\mathcal{S}_{\alpha}\left(t_{1}-s\right)\right]\left(\sigma^{*} x\right)(s) d s\right\|^{2} \\
\leq & \frac{14 \operatorname{Tr}(Q)(1-q) \Psi_{\sigma}(R)}{2 \alpha-1-q} \sup _{s \in\left[0, t_{1}-\varepsilon\right]}\left\|\mathcal{S}_{\alpha}\left(t_{2}-s\right)-\mathcal{S}_{\alpha}\left(t_{1}-s\right)\right\|^{2}\left\|\xi_{\sigma}\right\|_{L^{\frac{1}{q}}} \\
& \times\left(t_{1}^{\frac{2 \alpha-1-q_{2}}{1-q}}-\varepsilon^{\frac{2 \alpha-1-q}{1-q}}\right)^{1-q} \\
& +14 \operatorname{Tr}(Q)\left(\frac{2 M}{\Gamma(\alpha)}\right)^{2} \Psi_{\sigma}(R)\left\|\xi_{\sigma}\right\|_{L^{\frac{1}{q}}}\left(\frac{1-q}{2 \alpha-1-q}\right)^{2 \alpha-1-q} \varepsilon^{2 \alpha-1-q} \\
\rightarrow & 0 \quad\left(t_{2}-t_{1} \rightarrow 0 \text { and } \varepsilon \rightarrow 0\right) .
\end{aligned}
$$

Overall, we have $I_{i} \rightarrow 0$ as $t_{2}-t_{1} \rightarrow 0$ and $\varepsilon \rightarrow 0$, which means $F_{1}\left(B_{r}(\delta)\right)$ is equicontinuous. Therefore, an application of Arzela-Ascoli theorem justifies the precompactness of $F_{\delta}\left(B_{r}(\delta)\right)$. Thus, by Schauder fixed point theorem, $F_{\delta}$ has at least a fixed point $\bar{x} \in B_{R}(\delta)$, i.e.,

$$
\begin{aligned}
\bar{x}(t)= & \mathcal{T}_{\alpha}(t) g^{*}(\bar{x})+\int_{0}^{t}(t-s)^{\alpha-1} \mathcal{S}_{\alpha}(t-s)\left[\left(f^{*} \bar{x}\right)(s)+B u_{\varepsilon}^{*}(s, \bar{x})\right] d s \\
& +\int_{0}^{t}(t-s)^{\alpha-1} \mathcal{S}_{\alpha}(t-s)\left(\sigma^{*} \bar{x}\right)(s) d W(s), \quad t \in[\delta, b] .
\end{aligned}
$$

Set

$$
\begin{aligned}
\bar{y}(t)= & \mathcal{T}_{\alpha}(t) g^{*}(\bar{x})+\int_{0}^{t}(t-s)^{\alpha-1} \mathcal{S}_{\alpha}(t-s)\left[\left(f^{*} \bar{x}\right)(s)+B u_{\varepsilon}^{*}(s, \bar{x})\right] d s \\
& +\int_{0}^{t}(t-s)^{\alpha-1} \mathcal{S}_{\alpha}(t-s)\left(\sigma^{*} \bar{x}\right)(s) d W(s), \quad t \in[0, b] .
\end{aligned}
$$

Clearly, $\bar{x}(t)=\bar{y}(t)$ for $t \in[\delta, b]$. From the definitions of $f^{*}, \sigma^{*}$ and $g^{*}$, it follows immediately that

$$
\begin{aligned}
\bar{y}(t)= & \mathcal{T}_{\alpha}(t) g(\bar{y})+\int_{0}^{t}(t-s)^{\alpha-1} \mathcal{S}_{\alpha}(t-s)\left[f(s, \bar{y}(s))+B u_{\varepsilon}(s, \bar{y})\right] d s \\
& +\int_{0}^{t}(t-s)^{\alpha-1} \mathcal{S}_{\alpha}(t-s) \sigma(s, \bar{y}(s)) d W(s), \quad t \in[0, b] .
\end{aligned}
$$

that is, $\bar{y}$ is a mild solution of system (1.1) in $B_{R}$.

This completes the proof of Lemma 2.13 . 
For each $\delta \in(0, b)$ and arbitrary $x \in \mathcal{H}\left([0, b], L^{2}(\Omega, \mathbb{H})\right)$, write

$$
\left(\mathcal{L}_{\delta} x\right)(t)= \begin{cases}x(\delta), & t \in[0, \delta], \\ x(t), & t \in[\delta, b]\end{cases}
$$

and

$$
\begin{array}{ll}
f_{\delta}(t, x(t))=f\left(t,\left(\mathcal{L}_{\delta} x\right)(t)\right), & t \in[0, b], \\
\sigma_{\delta}(t, x(t))=\sigma\left(t,\left(\mathcal{L}_{\delta} x\right)(t)\right), & t \in[0, b], \\
h_{\delta}(t, x(t))=h\left(t,\left(\mathcal{L}_{\delta} x\right)(t)\right), & t \in[0, b],
\end{array}
$$

It is easy to see that $f_{\delta}, \sigma_{\delta}$, and $h_{\delta}$ defined above satisfy condition (H5), thus we obtain

Lemma 2.14 Assume that $-A$ generates a compact $C_{0}$-semigroup $T(t)(t \geq 0)$ of uniformly bounded operators in a Hilbert space $\mathbb{H}$. Let Assumptions $(\mathrm{H} 1)-(\mathrm{H} 4)$ hold. Then the following nonlocal problem:

$$
\left\{\begin{aligned}
{ }^{c} D_{t}^{\alpha} x(t)+A x(t)= & f_{\delta}(t, x(t))+\sigma_{\delta}(t, x(t)) \frac{d W(t)}{d t} \\
& +B u_{\varepsilon}\left(t, \mathcal{L}_{\delta} x\right), \quad t \in[0, b]
\end{aligned}\right.
$$

has at least one mild solution in $B_{R}$ provided that there exists a positive constant $R$ such that (2.17) is satisfied.

\section{Main results}

In this section, we shall discuss the finite-approximate controllability of the fractional stochastic dynamical control system (1.1) by using the approximation techniques and a diagonal argument.

Theorem 3.1 Assume that-A generates a compact $C_{0}$-semigroup $T(t)(t \geq 0)$ of uniformly bounded operators in a Hilbert space $\mathbb{H}$. If Assumptions $(\mathrm{H} 1)-(\mathrm{H} 4)$ are satisfied, then fractional stochastic control system with nonlocal conditions (1.1) has at least one mild solution in $\mathcal{H}\left([0, b], L^{2}(\Omega, \mathbb{H})\right)$ provided that there exists a positive constant $R$ such that $(2.17)$ is satisfied.

Proof To begin with, let $\left\{\delta_{n}: n \in \mathbb{N}\right\}$ be a decreasing sequence in $(0, b)$ with $\lim _{n \rightarrow \infty} \delta_{n}=0$. For every $n$, according to Lemma 2.14 , we claim that the following system:

$$
\left\{\begin{aligned}
{ }^{c} D_{t}^{\alpha} x(t)+A x(t)= & f_{\delta_{n}}(t, x(t))+\sigma_{\delta_{n}}(t, x(t)) \frac{d W(t)}{d t} \\
& +B u_{\varepsilon}\left(t,\left(\mathcal{L}_{\delta_{n}} x\right)(t)\right), \quad t \in[0, b], \\
x(0)=\int_{0}^{b} h_{\delta_{n}}(s, x(s)) d s &
\end{aligned}\right.
$$


has a mild solution $x_{n} \in B_{R}$ if constant $R$ satisfies (2.17), which is expressed by

$$
\begin{aligned}
x_{n}(t)= & \mathcal{T}_{\alpha}(t) \int_{0}^{b} h_{\delta_{n}}(s, x(s)) d s \\
& +\int_{0}^{t}(t-s)^{\alpha-1} \mathcal{S}_{\alpha}(t-s)\left[\left(f_{\delta_{n}}\left(s, x_{n}(s)\right)+B u_{\varepsilon}\left(s, \mathcal{L}_{\delta_{n}} x_{n}\right)\right] d s\right. \\
& +\int_{0}^{t}(t-s)^{\alpha-1} \mathcal{S}_{\alpha}(t-s)\left(\sigma_{\delta_{n}}\left(s, x_{n}(s)\right) d W(s), \quad t \in[0, b] .\right.
\end{aligned}
$$

Let

$$
v_{n}(t)= \begin{cases}x_{n}\left(\delta_{n}\right), & t \in\left[0, \delta_{n}\right], \\ x_{n}(t), & t \in\left[\delta_{n}, b\right],\end{cases}
$$

then $v_{n} \in B_{R}$. In view of the definitions of $f_{\delta_{n}}, \sigma_{\delta_{n}}$, and $h_{\delta_{n}}$, we conclude that

$$
\begin{aligned}
x_{n}(t)= & \mathcal{T}_{\alpha}(t) \int_{0}^{b} h\left(s, v_{n}(s)\right) d s \\
& +\int_{0}^{t}(t-s)^{\alpha-1} \mathcal{S}_{\alpha}(t-s)\left[\left(f\left(s, v_{n}(s)\right)+B u_{\varepsilon}\left(s, v_{n}\right)\right)\right] d s \\
& +\int_{0}^{t}(t-s)^{\alpha-1} \mathcal{S}_{\alpha}(t-s)\left(\sigma\left(s, v_{n}(s)\right) d W(s), \quad t \in[0, b] .\right.
\end{aligned}
$$

Next, we will show that the set $\left\{x_{n}: n \in \mathbb{N}\right\}$ is precompact in $\mathcal{H}\left([0, b], L^{2}(\Omega, \mathbb{H})\right)$. For this purpose, we introduce the following definition:

$$
\begin{aligned}
\rho_{n}(t)= & \mathcal{T}_{\alpha}(t) \int_{0}^{b} h\left(s, v_{n}(s)\right) d s, \quad t \in[0, b], \\
\varphi_{n}(t)= & \int_{0}^{t}(t-s)^{\alpha-1} \mathcal{S}_{\alpha}(t-s)\left[f\left(s, v_{n}(s)+B u_{\varepsilon}\left(s, v_{n}\right)\right)\right] d s \\
& +\int_{0}^{t}(t-s)^{\alpha-1} \mathcal{S}_{\alpha}(t-s) \sigma\left(s, v_{n}(s)\right) d W(s), \quad t \in[0, b], t \in[0, b] .
\end{aligned}
$$

Therefore, we only need to show that the sets $\left\{\rho_{n}: n \in \mathbb{N}\right\}$ and $\left\{\varphi_{n}: n \in \mathbb{N}\right\}$ are precompact in $\mathcal{H}\left([0, b], L^{2}(\Omega, \mathbb{H})\right)$.

From the expression of $v_{n}(t)$, we know that $v_{n} \in B_{R}$. This implies that (H1)-(H3) hold for $f\left(s, v_{n}(s)\right), \sigma\left(s, v_{n}(s)\right)$ and $h\left(s, v_{n}(s)\right)$. Moreover, $u_{\varepsilon}\left(s, v_{n}\right)$ satisfies the estimates (i) and (ii) in Lemma 2.12. Hence, it is not difficult to prove that the set $\left\{\varphi_{n}: n \in \mathbb{N}\right\}$ is precompact in $\mathcal{H}\left([0, b], L^{2}(\Omega, \mathbb{H})\right)$ by the arguments similar to those in the proof of Lemma 2.13. In the sequel, we will show that the set $\left\{\rho_{n}: n \in \mathbb{N}\right\}$ is also precompact in $\mathcal{H}\left([0, b], L^{2}(\Omega, \mathbb{H})\right)$. In fact, we only need to prove that the set $\left\{\int_{0}^{b} h\left(s, v_{n}(s)\right) d s: n \in \mathbb{N}\right\}$ is precompact in $\mathcal{H}\left([0, b], L^{2}(\Omega, \mathbb{H})\right)$.

Let $\left\{\eta_{n}: n \in \mathbb{N}\right\}$ be a decreasing sequence in $(0, b)$ such that $\lim _{n \rightarrow \infty} \eta_{n}=0$. For every $n \in \mathbb{N}$ and $t \in\left[\eta_{1}, b\right]$, define function $\omega_{n}:\left[\eta_{1}, b\right] \rightarrow \mathbb{H}$ by $\omega_{n}(t)=x_{n}(t)$. Note that $v_{n} \in B_{R}$ and hence, $\left\{\int_{0}^{b} h\left(s, v_{n}(s)\right) d s: n \in \mathbb{N}\right\}$ is bounded. Meanwhile, $\mathcal{T}_{\alpha}(t)$ is compact and norm-continuous for $t>0$, which implies that the set $\left\{\mathcal{T}_{\alpha}(t) \int_{0}^{b} h\left(s, v_{n}(s)\right) d s: n \in \mathbb{N}\right\}$ 
is precompact in $\mathbb{H}$ for any $t \in\left[\eta_{1}, b\right]$ and $\left\{\mathcal{T}_{\alpha}(\cdot) \int_{0}^{b} h\left(s, v_{n}(s)\right) d s: n \in \mathbb{N}\right\}$ is equicontinuous. By Arzela-Ascoli theorem, we conclude that $\left\{\mathcal{T}_{\alpha}(\cdot) \int_{0}^{b} h\left(s, v_{n}(s)\right) d s: n \in \mathbb{N}\right\}$ is precompact in $\mathcal{H}\left(\left[\eta_{1}, b\right], L^{2}(\Omega, \mathbb{H})\right)$. Combining this with the fact that $\left\{\varphi_{n}: n \in \mathbb{N}\right\}$ is precompact in $\mathcal{H}\left([0, b], L^{2}(\Omega, \mathbb{H})\right)$, we claim that $\left\{\omega_{n}: n \in \mathbb{N}\right\}$ is precompact in $\mathcal{H}\left(\left[\eta_{1}, b\right], L^{2}(\Omega, \mathbb{H})\right)$. Hence, we can find a subsequence $\left\{x_{n}^{1}: n \in \mathbb{N}\right\} \subset\left\{x_{n}: n \in \mathbb{N}\right\}$ which is a Cauchy sequence in $\mathcal{H}\left(\left[\eta_{1}, b\right], L^{2}(\Omega, \mathbb{H})\right)$. In the same way, we can select a subsequence $\left\{x_{n}^{2}: n \in\right.$ $\mathbb{N}\} \subset\left\{x_{n}^{1}: n \in \mathbb{N}\right\}$ which is a Cauchy sequence in $\mathcal{H}\left(\left[\eta_{2}, b\right], L^{2}(\Omega, \mathbb{H})\right)$. Repeating the above reasoning and applying a diagonal argument, we know that there exist a subsequence $\left\{x_{n}^{*}: n \in \mathbb{N}\right\} \subset\left\{x_{n}: n \in \mathbb{N}\right\}$ which is a Cauchy sequence in $\mathcal{H}\left(\left[\eta_{n}, b\right], L^{2}(\Omega, \mathbb{H})\right)$. Moreover, for every $t \in(0, b],\left\{x_{n}^{*}(t): n \in \mathbb{N}\right\}$ is a Cauchy sequence in $\mathbb{H}$. So there exists a continuous function $x^{*}:(0, b] \rightarrow L^{2}(\Omega, \mathbb{H})$ such that for each $\eta_{k}$,

$$
\lim _{n \rightarrow \infty} \max _{t \in\left[\eta_{k}, b\right]} \mathbb{E}\left\|x_{n}^{*}(t)-x^{*}(t)\right\|^{2}=0 .
$$

We further show that $\left\{g\left(x_{n}^{*}\right): n \in \mathbb{N}\right\}$ is a Cauchy sequence in $\mathbb{H}$. Let $\delta \in(0, b)$, then for any $x_{1}, x_{2} \in \mathcal{H}\left([0, b], L^{2}(\Omega, \mathbb{H})\right)$ with $x_{1}(t)=x_{2}(t), t \in[\delta, b]$, we have

$$
\mathbb{E}\left\|g\left(x_{1}\right)-g\left(x_{2}\right)\right\|^{2}=\mathbb{E}\left\|\int_{0}^{\delta}\left[h\left(s, x_{1}(s)\right) d s-h\left(s, x_{2}(s)\right)\right] d s\right\|^{2} \rightarrow 0 \quad(\delta \rightarrow 0) .
$$

Therefore, for $\forall \varepsilon>0$, there exists a positive constant $\delta_{0}<b$ such that

$$
\mathbb{E}\left\|g\left(x_{1}\right)-g\left(x_{2}\right)\right\|^{2}<\frac{\varepsilon}{16}
$$

for any $x_{1}, x_{2} \in \mathcal{H}\left([0, b], L^{2}(\Omega, \mathbb{H})\right)$ with $x_{1}(t)=x_{2}(t), t \in\left[\delta_{0}, b\right]$. Let $y(t)$ be the function defined by

$$
y(t)= \begin{cases}x^{*}\left(\delta_{0}\right), & t \in\left[0, \delta_{0}\right], \\ x^{*}(t), & t \in\left[\delta_{0}, b\right] .\end{cases}
$$

Clearly, $y \in \mathcal{H}\left([0, b], L^{2}(\Omega, \mathbb{H})\right)$. By (3.4) we have

$$
\lim _{n \rightarrow \infty} \max _{t \in\left[\delta_{0}, b\right]} \mathbb{E}\left\|x_{n}^{*}(t)-y(t)\right\|^{2}=0 .
$$

From the definition of $\mathcal{L}_{\delta}$, we can see easily that

$$
\lim _{n \rightarrow \infty}\left\|\mathcal{L}_{\delta_{0}} x_{n}^{*}-y\right\|_{\mathcal{H}}=0 .
$$

By the continuity of $g$, we can find a natural number $N$ such that

$$
\lim _{n \rightarrow \infty} \mathbb{E}\left\|g\left(\mathcal{L}_{\delta_{0}} x_{n}^{*}\right)-g(y)\right\|^{2}<\frac{\varepsilon}{16}, \quad n>N .
$$

Therefore, for any $m, n>N$, we have

$$
\begin{aligned}
\mathbb{E}\left\|g\left(x_{m}^{*}\right)-g\left(x_{n}^{*}\right)\right\|^{2} \leq & 4 \mathbb{E}\left\|g\left(x_{m}^{*}\right)-g\left(\mathcal{L}_{\delta_{0}} x_{m}^{*}\right)\right\|^{2}+4 \mathbb{E}\left\|g\left(\mathcal{L}_{\delta_{0}} x_{m}^{*}\right)-g(y)\right\|^{2} \\
& +4 \mathbb{E}\left\|g\left(\mathcal{L}_{\delta_{0}} x_{n}^{*}\right)-g(y)\right\|^{2}+4 \mathbb{E}\left\|g\left(x_{n}^{*}\right)-g\left(\mathcal{L}_{\delta_{0}} x_{n}^{*}\right)\right\|^{2} \\
< & \varepsilon
\end{aligned}
$$


This inequality implies that $\left\{g\left(x_{n}^{*}\right): n \in \mathbb{N}\right\}$ is a Cauchy sequence in $\mathbb{H}$, namely, $\left\{g\left(x_{n}\right): n \in\right.$ $\mathbb{N}$ \} is precompact in $\mathbb{H}$.

Finally, by (3.2), we can verify easily that

$$
\mathbb{E}\left\|g\left(x_{n}\right)-g\left(v_{n}\right)\right\|^{2} \rightarrow 0 \quad(n \rightarrow \infty)
$$

which implies that $\left\{\int_{0}^{b} h\left(s, v_{n}(s)\right) d s: n \in \mathbb{N}\right\}$ is precompact in $\mathbb{H}$.

So far we have proved that the set $\left\{x_{n}: n \in \mathbb{N}\right\} \subset \mathcal{H}\left([0, b], L^{2}(\Omega, \mathbb{H})\right)$ is precompact. Hence, there exist a subsequence of $\left\{x_{n}: n \in \mathbb{N}\right\}$ denoted again by $\left\{x_{n}: n \in \mathbb{N}\right\}$ and a function $x_{0} \in B_{R}$ such that

$$
\lim _{n \rightarrow \infty}\left\|x_{n}-x_{0}\right\|_{\mathcal{H}}^{2}=0
$$

From the definition of $v_{n}$ it follows that

$$
\begin{aligned}
\left\|v_{n}-x_{0}\right\|_{\mathcal{H}}^{2} & =\max _{t \in J} \mathbb{E}\left\|v_{n}(t)-x_{0}(t)\right\|^{2} \\
& \leq \max _{t \in\left[0, \delta_{n}\right]} \mathbb{E}\left\|x_{n}\left(\delta_{n}\right)-x_{0}(t)\right\|^{2}+\max _{t \in\left[\delta_{n}, b\right]} \mathbb{E}\left\|x_{n}(t)-x_{0}(t)\right\|^{2} \\
& \leq 2 \mathbb{E}\left\|x_{n}\left(\delta_{n}\right)-x_{0}\left(\delta_{n}\right)\right\|^{2}+2 \max _{t \in\left[0, \delta_{n}\right]} \mathbb{E}\left\|x_{0}\left(\delta_{n}\right)-x_{0}(t)\right\|^{2}+\left\|x_{n}-x_{0}\right\|_{\mathcal{H}}^{2} \\
& \leq\left\|x_{n}-x_{0}\right\|_{\mathcal{H}}^{2} \max _{t \in\left[0, \delta_{n}\right]} \mathbb{E}\left\|x_{0}\left(\delta_{n}\right)-x_{0}(t)\right\|^{2} \\
& \rightarrow 0 \quad(n \rightarrow \infty)
\end{aligned}
$$

By (3.6)-(3.7) and taking the limit as $n \rightarrow \infty$ in (3.3), one gets that

$$
\begin{aligned}
x_{0}(t)= & \mathcal{T}_{\alpha}(t) \int_{0}^{b} h\left(s, x_{0}(s)\right) d s \\
& +\int_{0}^{t}(t-s)^{\alpha-1} \mathcal{S}_{\alpha}(t-s)\left[\left(f\left(s, x_{0}(s)\right)+B u_{\varepsilon}\left(s, x_{0}\right)\right)\right] d s \\
& +\int_{0}^{t}(t-s)^{\alpha-1} \mathcal{S}_{\alpha}(t-s)\left(\sigma\left(s, x_{0}(s)\right) d W(s), \quad t \in[0, b] .\right.
\end{aligned}
$$

This means that $x_{0} \in \mathcal{H}\left([0, b], L^{2}(\Omega, \mathbb{H})\right)$ is a mild solution of system (1.1).

This completes the proof of Theorem 3.1.

Remark 3.2 Even if without control $u$ in the fractional nonlocal control system (1.1), Theorem 3.1 is still new.

Theorem 3.3 Suppose that the assumptions of Theorem 3.1 hold. Then the fractional control system (1.1) is finitely-approximately controllable on $[0, b]$.

Proof Let $x_{\varepsilon}$ be a mild solution of (1.1) in $B_{R}$. Then functional $J_{\varepsilon}\left(\phi, x_{\varepsilon}\right)$ has a unique critical point $\widehat{\phi}_{\varepsilon}$ such that $J_{\varepsilon}\left(\widehat{\phi}_{\varepsilon}, x_{\varepsilon}\right)=\min _{\phi \in \mathbb{H}} J_{\varepsilon}\left(\phi, x_{\varepsilon}\right)$. Therefore, for any $\psi \in \mathbb{H}$ and $\lambda \in \mathbb{R}$, we have

$$
J_{\varepsilon}\left(\widehat{\phi}_{\varepsilon}, x_{\varepsilon}\right) \leq J_{\varepsilon}\left(\widehat{\phi}_{\varepsilon}+\lambda \psi, x_{\varepsilon}\right) .
$$


By this inequality, for $\lambda>0$, we have

$$
\begin{aligned}
0 \leq & \frac{1}{\lambda}\left[J_{\varepsilon}\left(\widehat{\phi}_{\varepsilon}+\lambda \psi, x_{\varepsilon}\right)-J_{\varepsilon}\left(\widehat{\phi}_{\varepsilon}, x_{\varepsilon}\right)\right] \\
= & \int_{0}^{b}(b-s)^{\alpha-1} \mathbb{E}\left\langle B^{*} \mathcal{S}_{\alpha}^{*}(b-s) \widehat{\phi}_{\varepsilon}, B^{*} \mathcal{S}_{\alpha}^{*}(b-s) \psi\right\rangle d s \\
& +\frac{\lambda}{2} \int_{0}^{b}(b-s)^{\alpha-1} \mathbb{E}\left\|B^{*} \mathcal{S}_{\alpha}^{*}(b-s) \psi\right\|^{2} d s \\
& +\varepsilon \frac{\left(\mathbb{E}\left\|\left(I-\pi_{E}\right)\left(\widehat{\phi}_{\varepsilon}+\lambda \psi\right)\right\|^{2}\right)^{\frac{1}{2}}-\left(\mathbb{E}\left\|\left(I-\pi_{E}\right) \widehat{\phi}_{\varepsilon}\right\|^{2}\right)^{\frac{1}{2}}}{\lambda}-\mathbb{E}\left\langle\psi, p\left(x_{\varepsilon}\right)\right\rangle .
\end{aligned}
$$

Setting $\lambda \rightarrow 0^{+}$in the inequality above, we have

$$
\begin{aligned}
& \mathbb{E}\left\langle\psi, p\left(x_{\varepsilon}\right)\right\rangle-\int_{0}^{b}(b-s)^{\alpha-1} \mathbb{E}\left\langle B^{*} \mathcal{S}_{\alpha}^{*}(b-s) \widehat{\phi}_{\varepsilon}, B^{*} \mathcal{S}_{\alpha}^{*}(b-s) \psi\right\rangle d s \\
& \quad \leq \varepsilon\left(\mathbb{E}\left\|\left(I-\pi_{E}\right) \psi\right\|^{2}\right)^{\frac{1}{2}} .
\end{aligned}
$$

Similarly to the process above with $\lambda<0$, we get

$$
\begin{aligned}
& \left.|\mathbb{E}| \psi, p\left(x_{\varepsilon}\right)\right\rangle-\int_{0}^{b}(b-s)^{\alpha-1} \mathbb{E}\left|B^{*} \mathcal{S}_{\alpha}^{*}(b-s) \widehat{\phi}_{\varepsilon}, B^{*} \mathcal{S}_{\alpha}^{*}(b-s) \psi\right\rangle d s \mid \\
& \quad \leq \varepsilon\left(\mathbb{E}\left\|\left(I-\pi_{E}\right) \psi\right\|^{2}\right)^{\frac{1}{2}} .
\end{aligned}
$$

Since

$$
\begin{gathered}
\int_{0}^{b}(b-s)^{\alpha-1} \mathbb{E}\left|B^{*} \mathcal{S}_{\alpha}^{*}(b-s) \widehat{\phi}_{\varepsilon}, B^{*} \mathcal{S}_{\alpha}^{*}(b-s) \psi\right\rangle d s \\
=\int_{0}^{b}(b-s)^{\alpha-1} \mathbb{E}\left|\mathcal{S}_{\alpha}(b-s) B u_{\varepsilon}\left(s, x_{\varepsilon}\right), \psi\right\rangle d s,
\end{gathered}
$$

from the definition of $p\left(x_{\varepsilon}\right)$ and (3.8)-(3.9), for any $\psi \in \mathbb{H}$, it follows that

$$
\left|\mathbb{E}\left\langle x_{\varepsilon}(b)-x_{b}, \psi\right\rangle\right| \leq \varepsilon\left(\mathbb{E}\left\|\left(I-\pi_{E}\right) \psi\right\|^{2}\right)^{\frac{1}{2}}
$$

By this and the properties of the orthogonal projection, we have

$$
\begin{aligned}
& \mathbb{E}\left\|x_{\varepsilon}(b)-x_{b}\right\|^{2}<\varepsilon^{2}, \\
& \pi_{E} x_{\varepsilon}(b)=\pi_{E} x_{b} .
\end{aligned}
$$

Therefore, the fractional stochastic control system (1.1) is finitely-approximately controllable on $[0, b]$.

This completes the proof of Theorem 3.3.

Remark 3.4 If we take an abstract space $C([0, b], \mathbb{H})$ with norm $\|x\|_{C}=\max _{t \in[0, b]}\|x(t)\|$, and functional

$$
J_{\varepsilon}(\phi, x)=\frac{1}{2} \int_{0}^{b}(b-s)^{\alpha-1}\left\|B^{*} \mathcal{S}_{\alpha}^{*}(b-s) \phi\right\|^{2} d s+\varepsilon\left\|\left(I-\pi_{E}\right) \phi\right\|-\langle\phi, p(x)\rangle,
$$


where

$$
p(x)=x_{b}-\left(\mathcal{T}_{\alpha}(b) \int_{0}^{b} h(s, x(s)) d s+\int_{0}^{b}(b-s)^{\alpha-1} \mathcal{S}_{\alpha}(b-s) f(s, x(x)) d s\right),
$$

the methods used in this paper can be applied to study the finite-approximate controllability of deterministic systems

$$
\left\{\begin{array}{l}
{ }^{c} D_{t}^{\alpha} x(t)+A x(t)=f(t, x(t))+B u(t), \quad t \in[0, b] \\
x(0)=\int_{0}^{b} h(s, x(s)) d s .
\end{array}\right.
$$

The corresponding results that appear are also new.

\section{Application}

To illustrate the main result, we consider the following fractional stochastic control system:

$$
\left\{\begin{aligned}
& \frac{\partial^{\frac{2}{3}}}{\partial t^{\frac{2}{3}}} x(z, t)-\frac{\partial^{2} x(z, t)}{\partial z^{2}}=\left(\frac{t x(z, t)}{2(1+|x(z, t)|)}+x(z, t)\right)+\left(e^{t} x(z, t)+\sin x(z, t)\right) \frac{d W(t)}{d t} \\
&+u(z, t), \quad t \in[0, b], z \in[0,1] \\
& x(0, t)=x(1, t), \quad t \in[0, b], \\
& x(z, 0)=\int_{0}^{b} s^{2} \sin \left(\frac{x(z, s)}{s}\right) d s, \quad z \in[0,1]
\end{aligned}\right.
$$

where $W(t)$ is a standard one-dimensional Brownian motion defined on the filtered probability space $\left(\Omega, \mathcal{F},\left\{\mathcal{F}_{t}\right\}_{t \geq 0}, P\right)$. To write the above system (4.1) into the abstract form of (1.1), let $\mathbb{H}=\mathbb{E}=\mathbb{U}=L^{2}[0,1]$ with the norm $\|\cdot\|$. Define the operator $A: D(A) \subset X \rightarrow \mathbb{H}$ by

$$
\begin{aligned}
& A v=-v^{\prime \prime}, \quad v \in D(A), \\
& D(A)=\left\{v \in \mathbb{H}, v, v^{\prime} \text { are absolutely continuous, } v^{\prime \prime} \in \mathbb{H}, v(0)=v(1)=0\right\} .
\end{aligned}
$$

We know that $-A$ generates a compact, analytic semigroup $T(t)(t \geq 0)$ in $\mathbb{H}$ and

$$
T(t) v=\sum_{n=1}^{\infty} e^{-n^{2} t}\left(v, v_{n}\right) v_{n},\|T(t)\| \leq e^{-t}, \quad t>0,
$$

where $v_{n}=\sqrt{2} \sin (n s), n=1,2, \ldots$ is the orthogonal set of eigenvectors in $A$.

For any $t \in[0, b]$, let $x(t)(z)=x(z, t), B u(t)(z)=u(z, t), f(t, x(t))(z)=\frac{t x(z, t)}{2(1+|x(z, t)|)}+x(z, t)$, $\sigma(t, x(t))(z)=e^{t} x(z, t)+\sin x(z, t), h(t, x(t))(z)=t^{2} \sin \left(\frac{x(z, t)}{t}\right)$. Then problem (4.1) can be rewritten in the abstract form of (1.1). From the definitions of nonlinear terms $f, \sigma$, and $h$, we can easily verify that Aassumptions (H1)-(H3) hold with $\xi_{f}(t)=\frac{t^{2}}{2}+2, \xi_{\sigma}(t)=2\left(e^{2 t}+1\right)$, $\xi_{h}(t)=t^{2}$, and $\Psi_{f}(s)=\Psi_{\sigma}(s)=\Psi_{h}(s)=s$. It can be easily seen that the deterministic linear fractional control system corresponding to (4.1) is approximately controllable on $[0, b]$ (see [28]). Hence, by Theorem 3.1, system (4.1) has a mild solution provided that (2.17) holds. Also, all the conditions of Theorem 3.3 are satisfied, so we get that stochastic control system (4.1) is approximately controllable on $[0, b]$. 


\section{Acknowledgements}

The authors would like to thank the anonymous reviewers for their valuable comments and suggestions.

\section{Funding}

This work was supported by the National Natural Science Foundation of China (No. 11661071).

\section{Abbreviations}

Not applicable.

Availability of data and materials

Data sharing not applicable to this article as no datasets were generated or analyzed during the current study.

\section{Competing interests}

The authors declare that they have no competing interests.

\section{Authors' contributions}

YD and YL wrote the first draft of this manuscript, YD prepared the final version of the manuscript. All authors read and approved the final version of the manuscript.

\section{Publisher's Note}

Springer Nature remains neutral with regard to jurisdictional claims in published maps and institutional affiliations.

\section{Received: 15 May 2019 Accepted: 24 March 2020 Published online: 07 April 2020}

\section{References}

1. Byszewski, L.: Theorems about the existence and uniqueness of solutions of a semilinear evolution nonlocal Cauchy problem. J. Math. Anal. Appl. 162, 494-505 (1991)

2. Aizicovici, S., Gao, Y.: Functional differential equations with nonlocal initial conditions. J. Math. Anal. Appl. 10, 145-156 (1997)

3. Liu, J.H.: A remark on the mild solutions of non-local evolution equations. Semigroup Forum 66, 63-67 (2003)

4. Balachandran, K., Park, J.Y.: Nonlocal Cauchy problem for abstract fractional semilinear evolution equations. Nonlinear Anal. 71, 4471-4475 (2009)

5. Balachandran, K., Trujillo, J.J.: The nonlocal Cauchy problem for nonlinear fractional integro-differential equations in Banach spaces. Nonlinear Anal. 72, 4587-4593 (2010)

6. Zhou, Y., Jiao, F.: Nonlocal Cauchy problem for fractional evolution equations. Nonlinear Anal. 11, 4465-4475 (2010)

7. Zhou, Y., Jiao, F.: Existence of mild solutions for fractional neutral evolution equations. Comput. Math. Appl. 59, 1063-1077 (2010)

8. Diagana, T., Mophou, G.M., N'Guérékata, G.M.: On the existence of mild solutions to some semilinear fractional integro-differential equations. Electron. J. Qual. Theory Differ. Equ. 2010, 58 (2010)

9. Debbouche, A., Nieto, J.J.: Sobolev type fractional abstract evolution equations with nonlocal conditions and optimal multi-controls. Appl. Math. Comput. 245, 74-85 (2014)

10. Yang, M., Wang, Q.R.: Existence of mild solutions for a class of Hilfer fractional evolution equations with nonlocal conditions. Fract. Calc. Appl. Anal. 20, 679-705 (2017)

11. Chen, P.Y., Zhang, X.P., Li, Y.X.: Fractional non-autonomous evolution equation with nonlocal conditions. J. Pseudo-Differ. Oper. Appl. 10, 955-973 (2019)

12. Chen, P.Y., Li, Y.X.: A blow up alternative result for fractional nonautonomous evolution equation of Volterra type. Commun. Pure Appl. Anal. 17, 1975-1992 (2018)

13. Chen, P.Y., Zhang, X.P., Li, Y.X.: Study on fractional non-autonomous evolution equations with delay. Comput. Math. Appl. 73, 794-803 (2017)

14. Liang, J., Liu, J., Xiao, T.J.: Nonlocal Cauchy problems governed by compact operator families. Nonlinear Anal. 57, 183-189 (2004)

15. Mahmudov, N.I.: Finite-approximate controllability of fractional evolution equations: variational approach. Fract. Calc. Appl. Anal. 21, 919-936 (2018)

16. Dauer, J.P., Mahmudov, N.I.: Approximate controllability of semilinear functional equations in Hilbert spaces. J. Math. Anal. Appl. 273, 310-327 (2002)

17. Mahmudov, N.I.: Approximate controllability of evolution systems with nonlocal conditions. Nonlinear Anal. 68 , 536-546 (2008)

18. Sukavanam, N., Kumar, S.: Approximate controllability of fractional order semilinear delay systems. J. Optim. Theory Appl. 151, 373-384 (2011)

19. Sakthivel, R., Ren, Y., Mahmudov, N.I: On the approximate controllability of semilinear fractional differential systems. Comput. Math. Appl. 62, 1451-1459 (2011)

20. Mahmudov, N.I., Zorlu, S.: On the approximate controllability of fractional evolution equations with compact analytic semigroup. J. Comput. Appl. Math. 259, 194-204 (2014)

21. Ge, F.D., Zhou, H.C., Kou, C.H.: Approximate controllability of semilinear evolution equations of fractional order with nonlocal and impulsive conditions via an approximating technique. Appl. Math. Comput. 275, 107-120 (2016)

22. Tai, Z.X., Wang, X.C.: Controllability of fractional-order impulsive neutral functional infinite delay integro-differential systems in Banach spaces. Appl. Math. Lett. 22, 1760-1765 (2009)

23. Liang, J., Yang, H.: Controllability of fractional integro-differential evolution equations with nonlocal conditions. Appl. Math. Comput. 254, 20-29 (2015)

24. Sakthivel, R., Mahmudov, N.I., Nieto, J.J.: Controllability for a class of fractional-order neutral evolution control systems. Appl. Math. Comput. 218, 10334-10340 (2012) 
25. Yang, H., Agarwal, R.P., Liang, Y.: Controllability for a class of integro-differential evolution equations involving non-local initial conditions. Int. J. Control 90, 2567-2574 (2017)

26. Balasubramaniam, P., Tamilalagan, P.: The solvability and optimal controls for impulsive fractional stochastic integro-differential equations via resolvent operators. J. Optim. Theory Appl. 174, 139-155 (2017)

27. Mahmudov, N.I., Denker, A.: On controllability of linear stochastic systems. Int. J. Control 73, 144-151 (2000)

28. Sakthivel, R., Suganya, S., Anthoni, S.M.: Approximate controllability of fractional stochastic evolution equations. Comput. Math. Appl. 63, 660-668 (2012)

29. Dauer, J.P., Mahmudov, N...: Controllability of stochastic semilinear functional differential equations in Hilbert spaces, J. Math. Anal. Appl. 290, 373-394 (2004)

30. Muthukumar, P., Rajivganthi, C.: Approximate controllability of fractional order neutral stochastic integro-differential system with nonlocal conditions and infinite delay. Taiwan. J. Math. 17, 1693-1713 (2013)

31. Farahi, S., Guendouzi, T.: Approximate controllability of fractional neutral stochastic evolution equations with nonlocal conditions. Results Math. 65, 501-521 (2014)

32. Chen, P.Y., Zhang, X.P., Li, Y.X.: Approximate controllability of non-autonomous evolution system with nonlocal conditions. J. Dyn. Control Syst. 26, 1-16 (2020)

33. El-Borai, M.M., Moustafa, O.L., Ahmed, H.M.: Asymptotic stability of some stochastic evolution equations. Appl. Math. Comput. 144, 273-286 (2003)

34. Chen, P.Y., Zhang, X.P., Li, Y.X.: Nonlocal problem for fractional stochastic evolution equations with solution operators. Fract. Calc. Appl. Anal. 19, 1507-1526 (2016)

35. Chen, P.Y., Li, Y.X., Zhang, X.P.: On the initial value problem of fractional stochastic evolution equations in Hilbert spaces. Commun. Pure Appl. Anal. 14, 1817-1840 (2015)

36. Taniguchi, T., Liu, K., Truman, A.: Existence, uniqueness and asymptotic behavior of mild solutions to stochastic functional differential equations in Hilbert spaces. J. Differ. Equ. 181, 72-91 (2002)

37. Mao, X: Stochastic Differential Equations and Their Applications. Horwood, Chichester (1997)

38. Sakthivel, R., Revathi, P., Ren, Y.: Existence of solutions for nonlinear fractional stochastic differential equations. Nonlinear Anal. 81, 70-86 (2013)

39. Da Prato, G., Zabczyk, J.: Stochastic Equations in Infinite Dimensions. Cambridge University Press, Cambridge (1992)

40. Curtain, R.F., Falb, P.L.: Stochastic differential equations in Hilbert space. J. Differ. Equ. 10, 412-430 (1971)

41. Kilbas, A.A., Srivastava, H.M., Trujillo, J.J.: Theory and Applications of Fractional Differential Equations. North-Holland Mathematics Studies. Elsevier, Amsterdam (2006)

\section{Submit your manuscript to a SpringerOpen ${ }^{\circ}$ journal and benefit from:}

- Convenient online submission

- Rigorous peer review

- Open access: articles freely available online

- High visibility within the field

- Retaining the copyright to your article

Submit your next manuscript at $\gg$ springeropen.com 